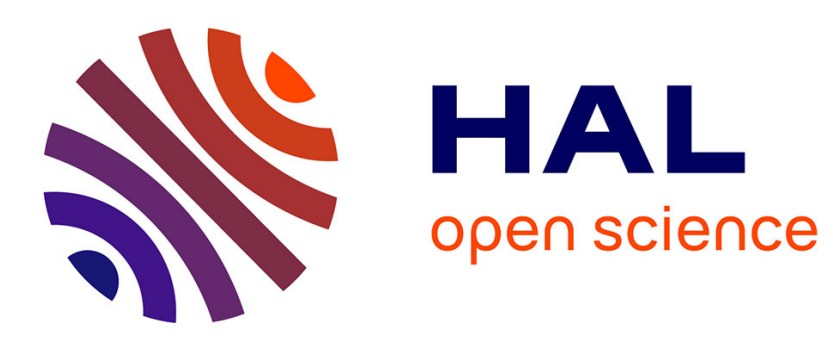

\title{
Dynamics and invariants of the perceived velocity gradient tensor in homogeneous and isotropic turbulence
}

Ping-Fan Yang, Alain Pumir, Haitao Xu

\section{To cite this version:}

Ping-Fan Yang, Alain Pumir, Haitao Xu. Dynamics and invariants of the perceived velocity gradient tensor in homogeneous and isotropic turbulence. Journal of Fluid Mechanics, 2020, 897, 10.1017/jfm.2020.375 . hal-03010642

\section{HAL Id: hal-03010642 \\ https://hal.science/hal-03010642}

Submitted on 30 Nov 2020

HAL is a multi-disciplinary open access archive for the deposit and dissemination of scientific research documents, whether they are published or not. The documents may come from teaching and research institutions in France or abroad, or from public or private research centers.
L'archive ouverte pluridisciplinaire HAL, est destinée au dépôt et à la diffusion de documents scientifiques de niveau recherche, publiés ou non, émanant des établissements d'enseignement et de recherche français ou étrangers, des laboratoires publics ou privés. 


\title{
Dynamics and invariants of the perceived velocity gradient tensor in homogeneous and isotropic turbulence
}

\author{
By Ping-Fan Yang ${ }^{1}$, Alain Pumir $^{2,3}$, and Haitao $\mathbf{X u}^{4}$ \\ ${ }^{1}$ Center for Combustion Energy and Department of Energy and Power Engineering \\ Tsinghua University, Beijing, 100084, China \\ ${ }^{2}$ Laboratoire de Physique, Ecole Normale Supérieure de Lyon, CNRS \\ Université de Lyon, Lyon, F-69007 France \\ ${ }^{3}$ Max Planck Institute for Dynamics and Self-Organization, Am Fassberg 17, Göttingen, \\ D-37077, Germany \\ ${ }^{4}$ Center for Combustion Energy and School of Aerospace Engineering \\ Tsinghua University, Beijing, 100084, China
}

(Received 22 December 2019)

The perceived velocity gradient tensor (PVGT), constructed from four fluid tracers forming regular tetrads of size $R_{0}$, provides a natural way to study the structure of velocity fluctuations and its dependence on the spatial scale. It generalizes and shares qualitatively many essential properties with the true velocity gradient tensor. Here, we establish the evolution equation of the PVGT, and in the case of a homogeneous and isotropic incompressible turbulent flow, we analyze the dynamics of the PVGT using its second and third order invariants. We show that the the second order invariants can be expressed solely in terms of the usual second order velocity structure functions, while the third order invariants is expressible as a combination of the third order velocity structure function, and of a less known three-point velocity correlation function. Exact relations between the second moments of strain and vorticity, as well as enstrophy production and the third moments of the strain, are derived. These relations, valid for all values of $R_{0}$, reduce when $R_{0}$ is in the dissipation range to classical results for the velocity gradient tensor, and generalize them when $R_{0}$ is in the inertial range. With the help of these relations, we quantify the importance of the various terms, such as vortex stretching, as a function of the spatial scale $R_{0}$. Our analysis, which is supported by the results of direct numerical simulations of turbulent flows in the Reynolds number range $100 \leqslant R_{\lambda} \leqslant 610$, allows us to demonstrate that strain prevails over vorticity when $R_{0}$ is in the inertial range of scales.

\section{Introduction}

The challenge to describe the physics of turbulent flows comes not only from the wide range of scales involved, but also from the spatial organization of the flow, which is responsible for the coupling between scales (Pope 2000; Frisch 1995; Monin \& Yaglom 1975). One manifestation of this complex structure is the emergence of tubes, where the magnitude of the vorticity vector $\boldsymbol{\omega}=\nabla \times \mathbf{U}$, is very high (Siggia 1981; Jimenez et al. 1992; Douady et al. 1991; Ishihara et al. 2007; Buaria et al. 2019). The amplification of vorticity results from its nonlinear coupling with the rate of strain tensor, $\mathbf{s}=\frac{1}{2}\left(\mathbf{m}+\mathbf{m}^{\mathbf{T}}\right)$, where $\mathbf{m}=\nabla \mathbf{U}$ is the velocity gradient tensor (Frisch 1995; Tsinober 2009). 
Much of the experimental investigation of turbulent flows has relied on the investigation of the velocity structure functions, defined as the moments of the difference between the component of flow velocity, $\mathbf{U}$, at two spatial points separated by a distance $\mathbf{x}$ along a spatial direction (say $x): D_{n}(x)=\left\langle(U(x)-U(0))^{n}\right\rangle$. While this quantity, defined with the help of two spatial points, is accessible from wind tunnel experiments (Comte-Bellot \& Corrsin 1966; Pope 2000; Bodenschatz et al. 2014) and provides a very useful characterisation of the scaling properties of the flow, it does not provides much information on the structural aspects of the velocity field. This deficiency is particularly important in the context of modeling the energy flux acting at small scales below the filtering scale in a Large Eddy Simulation approach (Borue \& Orszag 1998; Tao et al. 2002; Van der Bos et al. 2002; Meneveau 2011; Johnson \& Meneveau 2018). A possible approach to study simultaneously the structural and the scaling aspects of turbulence consists in considering the velocity at four points separated by a distance $R_{0}$ forming a regular tetrad (Chertkov et al. 1999). How such a tetrad deforms as the fluid particles move with the flow reveals interesting properties of the flow (Pumir et al. 2000; Biferale et al. 2005; Naso \& Pumir 2005; Xu et al. 2008; Hackl et al. 2011; Meneveau 2011; Xu et al. 2011; Naso \& Godeferd 2012; Devenish 2013; Naso 2019). Here, we focus on the perceived velocity gradient tensor (PVGT), M, obtained from the velocity differences over the distance between the four points defining the tetrad. The PVGT can be viewed as an extension of the velocity gradient tensor to length scales beyond the dissipation range (Chevillard \& Meneveau 2006; Meneveau 2011; Jucha et al. 2014; Xu et al. 2016; Johnson \& Meneveau 2016). Other attempts to study the velocity gradient beyond the dissipative scale include the velocity gradient coarse-grained over a spherical volume following a fluid particle trajectory (Meneveau \& Lund 1994), and the velocity gradient obtained from the velocities of fluid particles within a sphere centered at a target fluid particle (Lüthi et al. 2007). As we stress in this work, the study of the PVGT provides some information on the relative role of vorticity and strain as a function of scales, and also on their dynamics.

As shown in, e.g., Pumir et al. (2013), strong similarities exist between the properties of the PVGT and those of the true velocity gradient $\mathbf{m}$. Nonetheless, there are important differences between the two quantities. One of them comes from the incompressibility condition, which is not satisfied by $\mathbf{M}: \operatorname{tr}(\mathbf{M}) \neq 0$, except in the limit $R_{0} \rightarrow 0$, where $\mathbf{M}$ reduces to $\mathbf{m}$. This leads to quantitative differences in the properties of $\mathbf{m}$ and $\mathbf{M}$, which we analyze in this work.

Specifically, we decompose the PVGT as $\mathbf{M}=\mathbf{S}+\mathbf{W}+\frac{1}{3} \operatorname{tr}(\mathbf{M}) \mathbf{I}$, where $\mathbf{S}$ and $\mathbf{W}$ are the symmetric and antisymmetric parts of $\mathbf{M}$, respectively, and $\mathbf{I}$ is the identity tensor. We establish here the evolution equations for $\mathbf{M}, \mathbf{S}$ and $\mathbf{W}$. The equations for the quadratic invariants of $\mathbf{M}, \operatorname{tr}\left(\mathbf{S}^{2}\right)$ and $\operatorname{tr}\left(\mathbf{W}^{2}\right)$, differ from the corresponding invariants of $\mathbf{m}$ via terms involving the traces of powers of $\mathbf{M}$. In the case of homogeneous, isotropic turbulence in incompressible flows, the averaged values of $\operatorname{tr}\left(\mathbf{m}^{2}\right)$ and $\operatorname{tr}\left(\mathbf{m}^{3}\right)$ are exactly 0 (Betchov 1956). In fact, these relations allow one to express the second and third invariants of $\mathbf{m}$ in terms of $\left\langle\operatorname{tr}\left(\mathbf{s}^{2}\right)\right\rangle$ and $\left\langle\operatorname{tr}\left(\mathbf{s}^{3}\right)\right\rangle$ only. The deviation from the incompressibility $(\operatorname{tr}(\mathbf{M}) \neq 0)$ makes the situation more complicated for the PVGT. In this work, we generalize the exact relations obtained in Betchov (1956) to the PVGT, and together with the dynamic equations for $\mathbf{M}$, we discuss quantitatively the production of strain rate and vorticity. Overall, we find that strain rate prevails over vorticity in the inertial range.

This work is organized as follows. In Section 2, we recall the definition of $\mathbf{M}$, and derive its evolution equation from the Navier-Stokes equations. Section 3 generalizes the properties of the second and third moments of the true velocity gradient, $\mathbf{m}$, to the PVGT, $\mathbf{M}$, and provides exact expressions for all the quantities involved, in the spirit of Betchov 
(1956). Whereas our analysis relates most of these moments to the well-documented twopoint longitudinal structure functions of the second and third order Frisch (1995), the vortex stretching term also involves the genuine three-point correlation function, with 3 points on an equilateral triangle. In Section 4, we express the correlations involving the PVGT, M, and the fluid acceleration, appearing in the dynamics of the second and third moments of $\mathbf{M}$, in terms of the two-point structure function $D_{n}(r)$. Last, with the help of direct numerical simulation (DNS) data at several Reynolds numbers, we analyze in Section 5 the various terms in the equations for strain and vorticity production, and show the prevalence of strain over vorticity production in the inertial range. Finally, Section 6 presents our concluding remarks.

\section{The perceived-velocity gradient tensor}

In this section, we discuss the definition of the PVGT $\mathbf{M}$ based on regular tetrads, defined by a set of 4 points distant from each other by a size $R_{0}$. We also derive the equation of evolution for $\mathbf{M}$.

\subsection{Elementary construction of the perceived velocity gradient tensor}

We first introduce the convention used in this work. The construction of the PVGT used here closely follows previous work (Xu et al. 2011; Pumir et al. 2013).

Consider four fluid particles forming a regular tetrahedron in a homogeneous and isotropic flow, i.e., the mutual distances between any two of the four points are $R_{0}$. Denoting the positions and velocities of the four points in the laboratory frame by $\mathbf{X}^{\alpha}$ and $\mathbf{U}^{\alpha},(\alpha=1,2,3,4)$, respectively, we introduce the coordinates $\mathbf{x}^{\alpha}$ with respect to the center of mass: $\mathbf{x}^{\alpha}=\mathbf{X}^{\alpha}-\mathbf{X}^{0}$, where $\mathbf{X}^{0}=\frac{1}{4} \sum_{\alpha=1}^{4} \mathbf{X}^{\alpha}$, and the reduced velocity, $\mathbf{u}^{\alpha}, \mathbf{u}^{\alpha}=\mathbf{U}^{\alpha}-\mathbf{U}^{0}$, where $\mathbf{U}^{0}=\frac{1}{4} \sum_{\alpha=1}^{4} \mathbf{U}^{\alpha}$. The perceived velocity gradient tensor $\mathbf{M}$, based on the four points of the tetrahedron, is defined by:

$$
x_{j}^{\alpha} M_{j i}=u_{i}^{\alpha} \quad \text { for } \quad \alpha=1,2,3,4,
$$

or equivalently, after multiplying both terms of Eq. (2.1) by $x_{k}^{\alpha}$, and summing over $\alpha$ :

$$
M_{i j}=g_{i k}^{-1} \Xi_{k j},
$$

where the tensors $\mathbf{g}$ and $\boldsymbol{\Xi}$ are defined by:

$$
g_{i j} \equiv \sum_{\alpha=1}^{4} x_{i}^{\alpha} x_{j}^{\alpha} \quad \text { and } \quad \Xi_{i j} \equiv \sum_{\alpha=1}^{4} x_{i}^{\alpha} u_{j}^{\alpha} .
$$

When the tetrahedron is regular, the tensor $\mathbf{g}$ is isotropic: $g_{i j}=\frac{1}{3} \operatorname{tr}(\mathbf{g}) \delta_{i j}$, with trace $\operatorname{tr}(\mathbf{g})=\frac{3}{2} R_{0}^{2}$. Thus for a regular tetrahedron, Eq. (2.2) reduces to

$$
\mathbf{M}=\frac{2}{R_{0}^{2}} \mathbf{\Xi} .
$$

It is important to notice that, contrary to the velocity gradient tensor $\mathbf{m}$, which is always incompressible: $\operatorname{tr}(\mathbf{m})=0$, the PVGT is in general not incompressible: $\operatorname{tr}(\mathbf{M}) \neq 0$. This reflects the observation that at the level of the tetrad, the flow can locally lead to compression or expansion. In the following, we consider the trace of the tensor separately. We also consider the classical decomposition of the PVGT as a sum of its symmetric and anti-symmetric parts:

$$
M_{i j}=S_{i j}+W_{i j}+\frac{1}{3} \operatorname{tr}(\mathbf{M}) \delta_{i j}
$$


where $S_{i j}=\frac{1}{2}\left(M_{i j}+M_{j i}\right)-\frac{1}{3} \operatorname{tr}(\mathbf{M}) \delta_{i j}$ and $W_{i j}=\frac{1}{2}\left(M_{i j}-M_{j i}\right)$. The $\mathbf{S}$ and $\mathbf{W}$ terms in Eq.(2.5) describe the straining and rotational motions as perceived by the 4 points of the tetrad. The definition simplifies in the case of the true velocity gradient tensor to $\mathbf{m}=\mathbf{s}+\mathbf{w}$. Given the definitions used here, the PVGT $\mathbf{M}$ reduces to the velocity gradient tensor $\mathbf{m}$ when $R_{0} \rightarrow 0$. In practice, this limit is reached when $R_{0}$ decreases below the Kolmogorov's length scale $\eta=\left(\nu^{3} / \varepsilon\right)^{1 / 4}$, where $\varepsilon$ is the rate of kinetic energy dissipation per unit mass in the flow (Pumir et al. 2013).

\subsection{Evolution equation for the perceived velocity gradient tensor}

The equation of evolution for $\mathbf{M}$ can be derived from Eq. (2.1-2.3). Namely, taking the time derivatives of Eqs. (2.2) and (2.3) in the frame attached with $\mathbf{X}_{0}$ and moving with the center of mass velocity $\mathbf{U}_{0}$ yields

$$
\frac{d g_{i k}}{d t} M_{k j}+g_{i k} \frac{d M_{k j}}{d t}=\frac{d \Xi_{i j}}{d t}=\sum_{\alpha=1}^{4} u_{i}^{\alpha} u_{j}^{\alpha}+\sum_{\alpha=1}^{4} x_{i}^{\alpha} a_{j}^{\alpha},
$$

where $\mathbf{a}^{\alpha}$ is the accelerations of fluid particles relative to the center of mass, which is related to the acceleration in the laboratory frame $\mathbf{A}^{\alpha}$ by $\mathbf{a}^{\alpha}=\mathbf{A}^{\alpha}-\frac{1}{4} \sum_{\beta=1}^{4} \mathbf{A}^{\beta}$. The Navier-Stokes equation express that:

$$
\mathbf{A}^{\alpha}=\frac{d \mathbf{U}^{\alpha}}{d t}=-\nabla P^{\alpha}+\mathbf{F}^{\alpha}+\nu \nabla^{2} \mathbf{U}^{\alpha}
$$

where $\mathbf{F}$ is the external body force per unit mass.

In Eq. (2.6) we can rewrite the term $\sum_{\alpha=1}^{4} u_{i}^{\alpha} u_{j}^{\alpha}$ in terms of $\mathbf{M}$ and $\mathbf{g}$ as:

$$
\sum_{\alpha=1}^{4} u_{i}^{\alpha} u_{j}^{\alpha}=\sum_{\alpha=1}^{4} x_{k}^{\alpha} M_{k i} x_{n}^{\alpha} M_{n j}=M_{k i} g_{k n} M_{n j}=\mathbf{M}^{\mathrm{T}} \mathbf{g} \mathbf{M} .
$$

Differentiating $\mathbf{g}$, as defined by Eq. (2.3), with respect to time, leads to:

$$
\frac{d \mathbf{g}}{d t}=\mathbf{g} \mathbf{M}+\mathbf{M}^{\mathrm{T}} \mathbf{g}
$$

then substituting Eqs. (2.8) and (2.9) into Eq. (2.6) leads to, after some elementary algebra:

$$
\begin{aligned}
\frac{d \mathbf{M}}{d t} & =\mathbf{g}^{-1}\left[-\mathbf{g} \mathbf{M}^{2}-\mathbf{M}^{\mathrm{T}} \mathbf{g} \mathbf{M}+\mathbf{M}^{\mathrm{T}} \mathbf{g} \mathbf{M}+\mathbf{H} / \operatorname{tr}\left(\mathbf{g}^{-1}\right)\right]=-\mathbf{M}^{2}+\mathbf{\Pi} \mathbf{H} \\
& =-\mathbf{M}^{2}+\mathbf{\Pi} \mathbf{H}^{p}+\mathbf{\Pi} \mathbf{H}^{\nu}+\mathbf{\Pi} \mathbf{H}^{f}
\end{aligned}
$$

where the tensor $\boldsymbol{\Pi}=\frac{\mathbf{g}^{-1}}{\operatorname{tr}\left(\mathbf{g}^{-1}\right)}$ was introduced by Chertkov et al. (1999) and $\mathbf{H}$ is defined by:

$$
H_{i j}=\operatorname{tr}\left(\mathbf{g}^{-1}\right) \sum_{\alpha=1}^{4} x_{i}^{\alpha} a_{j}^{\alpha}=H_{i j}^{p}+H_{i j}^{\nu}+H_{i j}^{f},
$$

in which $H_{i j}^{p}, H_{i j}^{\nu}$, and $H_{i j}^{f}$ are the contributions to $H_{i j}$ from the components of $\mathbf{a}^{\alpha}$ corresponding to the pressure gradient, the viscous forces and the external forcing, see Eq. (2.7). Eq. (2.10) is very reminiscent of the evolution equation of $\mathbf{m}$ (Meneveau 2011):

$$
\frac{d m_{i j}}{d t}=-m_{i k} m_{k j}-\frac{\partial^{2} p}{\partial x_{i} \partial x_{j}}+\nu \frac{\partial^{2} m_{i j}}{\partial x_{k} \partial x_{k}}+\frac{\partial F_{i}}{\partial x_{j}},
$$

The strong resemblance between Eq. (2.10) and (2.12) is a direct consequence of the 
Navier-Stokes equations themselves. Namely, the quadratic nonlinear terms in Eqs. (2.10) and (2.12) are identical, and the terms $\boldsymbol{\Pi} \mathbf{H}^{p}, \boldsymbol{\Pi} \mathbf{H}^{\nu}$, and $\boldsymbol{\Pi} \mathbf{H}^{f}$ in Eqs. (2.10) represent the pressure hessian, the viscous diffusion, and the gradient of the external forcing in Eqs. (2.12), respectively.

To simplify the notation in the analysis, we denote throughout the rest of the text the trace of a tensor by a bar over the tensor:

$$
\overline{\mathbf{Y}} \equiv \operatorname{tr}(\mathbf{Y}) .
$$

Decomposing $\mathbf{M}$ as in Eq. (2.5), we readily obtain the equations for $\overline{\mathbf{M}}, \mathbf{S}$ and $\mathbf{W}$ :

$$
\begin{aligned}
\frac{d \overline{\mathbf{M}}}{d t} & =-\left(\overline{\mathbf{S}^{2}}+\overline{\mathbf{W}^{2}}+\frac{1}{3} \overline{\mathbf{M}}^{2}\right)+\overline{\mathbf{\Pi}} \\
\frac{d \mathbf{S}}{d t} & =-\mathbf{S}^{2}-\mathbf{W}^{2}-\frac{2}{3} \overline{\mathbf{M}} \mathbf{S}+\frac{1}{3}\left(\overline{\mathbf{S}^{2}}+\overline{\mathbf{W}^{2}}\right) \mathbf{I}+\frac{1}{2}\left[\mathbf{\Pi} \mathbf{H}+(\mathbf{\Pi} \mathbf{H})^{\mathrm{T}}\right]-\frac{1}{3} \overline{\mathbf{\Pi}} \mathbf{H} \mathbf{I} \\
\frac{d \mathbf{W}}{d t} & =-\mathbf{S W}-\mathbf{W} \mathbf{S}-\frac{2}{3} \overline{\mathbf{M}} \mathbf{W}+\frac{1}{2}\left[\mathbf{\Pi} \mathbf{H}-(\mathbf{\Pi} \mathbf{H})^{\mathrm{T}}\right]
\end{aligned}
$$

where we recall that $\mathbf{I}$ refers to the identity tensor. The evolution equations for $\mathbf{m}$ and $\mathbf{M}$ differ in several important ways. The first important difference is that $\overline{\mathbf{M}}$ is, in general, nonzero. This is made explicit in the decomposition Eq. (2.5), and results in terms involving $\overline{\mathbf{M}}$ in Eqs. (2.15) and (2.16). The second difference comes from the appearance of a pressure term $\overline{\mathbf{\Pi H}^{p}-\left(\mathbf{\Pi H}^{p}\right)^{\mathrm{T}}}$ in the equation for $\mathbf{W}$ through the term $\overline{\boldsymbol{\Pi} \mathbf{H}-(\boldsymbol{\Pi} \mathbf{H})^{\mathrm{T}}}$, while the pressure does not contribute to the equation for the antisymmetric part $\mathbf{w}$ of the velocity gradient tensor $\mathbf{m}$ :

$$
\frac{d \mathbf{w}}{d t}=-\mathbf{s w}-\mathbf{w s}+\nu \nabla^{2} \mathbf{w}+\frac{1}{2}\left[\nabla \mathbf{F}-(\nabla \mathbf{F})^{\mathrm{T}}\right] .
$$

The origin of this effect of pressure comes from the finite difference approximation, and the term $\overline{\mathbf{\Pi} \mathbf{H}^{p}-\left(\mathbf{\Pi H}^{p}\right)^{\mathrm{T}}}$ reduces to zero only in the $R_{0} \rightarrow 0$ limit. Last, the coupling between the evolution of $\mathbf{M}$ and the geometry, through the tensor $\mathbf{g}$ leads to the most significant difference. As a result of this coupling, the evolution of $\mathbf{M}$ is not determined by (2.10) alone, as the shape and size of the tetrads evolve along with $\mathbf{M}$. Taking into account these deformations is essential in the physics of the PVGT (Pumir et al. 2013). In particular, Eqs. (2.15) and (2.16) provide a way to investigate the production of strain rate and vorticity, and their dependence on scale. To quantify the production of vorticity and strain, we will particularly focus on the equations for the invariants $\left\langle\overline{\mathbf{S}^{2}}\right\rangle$ and $\left\langle\overline{\mathbf{W}^{2}}\right\rangle$, where the brackets $\langle\cdot\rangle$ denote an ensemble average over many tetrads with the same geometry in the flow. Straightforward algebraic manipulations lead to:

$$
\frac{1}{2} \frac{d\left\langle\overline{\mathbf{S}^{2}}\right\rangle}{d t}=-\left\langle\overline{\mathbf{S}^{3}}\right\rangle-\langle\overline{\mathbf{W S W}}\rangle-\frac{2}{3}\left\langle\overline{\mathbf{M}} \overline{\mathbf{S}^{2}}\right\rangle+\langle\overline{\mathbf{M H S}}\rangle,
$$

and

$$
\frac{1}{2} \frac{d\left\langle\overline{\mathbf{W}^{2}}\right\rangle}{d t}=-2\langle\overline{\mathbf{W S W}}\rangle-\frac{2}{3}\left\langle\overline{\mathbf{M}} \overline{\mathbf{W}^{2}}\right\rangle+\langle\overline{\mathbf{\Pi H W}}\rangle .
$$

Equation (2.19) reduces, for very small tetrads $R_{0} \ll \eta$, to the well known equation for the evolution of enstrophy:

$$
\frac{d\left\langle\frac{1}{2} \boldsymbol{\omega}^{2}\right\rangle}{d t}=-\frac{1}{4} \frac{d\left\langle\overline{\mathbf{w}^{2}}\right\rangle}{d t}=\langle\overline{\mathbf{w S w}}\rangle+\nu\left\langle\boldsymbol{\omega} \cdot \nabla^{2} \boldsymbol{\omega}\right\rangle+\langle\boldsymbol{\omega} \cdot \nabla \times \mathbf{F}\rangle,
$$

where the vorticity $\boldsymbol{\omega}$ is related to the antisymmetric part of $\mathbf{m}$ by $w_{i j}=-\frac{1}{2} \epsilon_{i j k} \omega_{k}$, 
where $\epsilon_{i j k}$ is the permutation tensor. We have made explicit use in Eq. (2.20) of the relation $\boldsymbol{\omega}^{2}=-2 \overline{\mathbf{w}^{2}}$, and we further notice that $\overline{\mathbf{w S w}}=\frac{1}{4} \boldsymbol{\omega} \cdot \mathbf{s} \cdot \boldsymbol{\omega}$.

The coupling between the PVGT and the geometry, i.e., terms $\langle\overline{\mathbf{\Pi H S}}\rangle$ and $\langle\overline{\mathbf{\Pi H W}}\rangle$, implies that the averages of the time derivatives of the quadratic invariants, $-\mathbf{W}^{2}$ and $\mathbf{S}^{2}$ over many identical tetrads in the flow, are not zero, even if the flow is statistically stationary: vorticity or strain can grow, as measured by following an initially regular Lagrangian tetrad of size $R_{0}$. This property is interesting on its own right, as it allows us to characterize enstrophy and strain production as a function of scale.

As it was the case for the velocity gradient tensor (Betchov 1956), a systematic analysis of the invariants of the PVGT, M, helps in the understanding of the dynamics of vorticity and strain rate, as we document in the following section.

\section{Betchov relations generalized to the PVGT}

In addition to the identity $\overline{\mathbf{m}} \equiv \operatorname{tr}(\mathbf{m})=0$, which simply results from incompressibility, it was established (Townsend 1951; Betchov 1956) that in homogeneous flows,

$$
\left\langle\overline{\mathbf{m}^{2}}\right\rangle=\left\langle\overline{\mathbf{m}^{3}}\right\rangle=0 .
$$

These equalities result from elementary algebraic manipulations, and lead to the following identities:

$$
\begin{aligned}
& \left\langle\overline{\mathbf{s}^{2}}\right\rangle=-\left\langle\overline{\mathbf{w}^{2}}\right\rangle=\frac{1}{2}\left\langle\boldsymbol{\omega}^{2}\right\rangle, \\
& \left\langle\overline{\mathbf{s}^{3}}\right\rangle=-3\langle\overline{\mathbf{w s w}}\rangle=-\frac{3}{4}\langle\boldsymbol{\omega} \cdot \mathbf{s} \cdot \boldsymbol{\omega}\rangle,
\end{aligned}
$$

Equation (3.2) connects the amplitudes of vorticity and the rate of strain, while Eq. (3.3), remarkably, relates the rate of generation of enstrophy, $\langle\boldsymbol{\omega} \cdot \mathbf{s} \cdot \boldsymbol{\omega}\rangle$, see Eq. (2.20), to the properties of the rate of strain. Namely, Eq. (3.3) expresses the mean rate of generation of enstrophy in terms of the eigenvalues of $\mathbf{s}, \lambda_{1}, \lambda_{2}$ and $\lambda_{3}$ (ordered such that $\lambda_{1} \geqslant \lambda_{2} \geqslant$ $\left.\lambda_{3}\right):\langle\boldsymbol{\omega} \cdot \mathbf{s} \cdot \boldsymbol{\omega}\rangle=-4\left\langle\lambda_{1} \lambda_{2} \lambda_{3}\right\rangle$. Enstrophy production implies that $\left\langle\lambda_{1} \lambda_{2} \lambda_{3}\right\rangle<0$, so the intermediate eigenvalue $\lambda_{2}$ is preferentially positive (Betchov 1956; Tsinober 2009).

In the rest of this section, assuming the flow is statistically homogeneous and isotropic, we will extend relations Eq. (3.2) and Eq. (3.3) to the PVGT M. The first step will be to establish relations between quantities $\overline{\mathbf{M}^{n}}$ for $n=2$ and 3, generalizing Eq. (3.1). We begin by noticing that for $n=1$, we return to Eq. (2.4) and (2.3), to obtain:

$$
\langle\overline{\mathbf{M}}\rangle=\frac{2}{R_{0}^{2}} \sum_{\alpha=1}^{4} x_{i}^{\alpha}\left\langle u_{i}^{\alpha}\right\rangle=0
$$

as a result of the homogeneous condition $\left\langle u_{i}^{\alpha}\right\rangle=0$ for any particle $\alpha(1 \leqslant \alpha \leqslant 4)$ and for any component $i(1 \leqslant i \leqslant 3)$.

\subsection{Second-order moments of $M$}

\subsubsection{Generalized Betchov relations for the second moments}

To simplify the notation, we denote the various second moments of $\mathbf{M}$ by $T_{2}^{p}(1 \leqslant p \leqslant$ 3), defined as:

$$
T_{2}^{1}=\left\langle\overline{\mathbf{M}^{2}}\right\rangle, \quad T_{2}^{2}=\left\langle\overline{\mathbf{M M}^{\mathrm{T}}}\right\rangle, \quad \text { and } T_{2}^{3}=\left\langle\overline{\mathbf{M}}^{2}\right\rangle .
$$

The invariants such as $\left\langle\overline{\mathbf{S}^{2}}\right\rangle$ and $\left\langle\overline{\mathbf{W}^{2}}\right\rangle$ can be simply deduced from the equalities:

$$
T_{2}^{1}=\left\langle\overline{\mathbf{S}^{2}}\right\rangle+\left\langle\overline{\mathbf{W}^{2}}\right\rangle+\frac{1}{3} T_{2}^{3}, \quad \text { and } \quad T_{2}^{2}=\left\langle\overline{\mathbf{S}^{2}}\right\rangle-\left\langle\overline{\mathbf{W}^{2}}\right\rangle+\frac{1}{3} T_{2}^{3}
$$


To evaluate $T_{2}^{p}$, we start with Eqs. (2.4) and (2.3). An elementary calculation leads to:

$$
\begin{aligned}
T_{2}^{1} & =\left\langle\frac{4}{R_{0}^{4}} \overline{\boldsymbol{\Xi}^{2}}\right\rangle=\left\langle\frac{4}{R_{0}^{4}}\left(\sum_{\alpha=1}^{4} x_{i}^{\alpha} u_{j}^{\alpha}\right)\left(\sum_{\beta=1}^{4} x_{j}^{\beta} u_{i}^{\beta}\right)\right\rangle \\
& =\frac{4}{R_{0}^{4}}\left(4 x_{i}^{1}\left\langle u_{i}^{1} u_{j}^{1}\right\rangle x_{j}^{1}+12 x_{i}^{1}\left\langle u_{i}^{2} u_{j}^{1}\right\rangle x_{j}^{2}\right) . \\
T_{2}^{2} & =\left\langle\frac{4}{R_{0}^{4}} \overline{\boldsymbol{\Xi}^{\mathrm{T}}}\right\rangle=\left\langle\frac{4}{R_{0}^{4}}\left(\frac{R_{0}^{2}}{2} \sum_{\alpha=1}^{4} u_{i}^{\alpha} u_{i}^{\alpha}\right)\right\rangle=\frac{8}{R_{0}^{2}}\left\langle u_{i}^{1} u_{i}^{1}\right\rangle, \\
T_{2}^{3} & =\left\langle\frac{4}{R_{0}^{4}} \overline{\boldsymbol{\Xi}}^{2}\right\rangle=\left\langle\frac{4}{R_{0}^{4}}\left(\sum_{\alpha=1}^{4} x_{i}^{\alpha} u_{i}^{\alpha}\right)\left(\sum_{\beta=1}^{4} x_{j}^{\beta} u_{j}^{\beta}\right)\right\rangle \\
& =\frac{4}{R_{0}^{4}}\left(4 x_{i}^{1}\left\langle u_{i}^{1} u_{j}^{1}\right\rangle x_{j}^{1}+12 x_{i}^{1}\left\langle u_{i}^{1} u_{j}^{2}\right\rangle x_{j}^{2}\right) .
\end{aligned}
$$

To obtain the expressions of $T_{2}^{p}$ in terms of the velocity correlations in the equations above, we used the symmetry between the vertices of a regular tetrahedron and the isotropy of the flow field. The second moments $T_{2}^{p}$ are therefore expressed in terms of the 2-point velocity correlation functions $\left\langle u_{i}^{1} u_{j}^{1}\right\rangle$ and $\left\langle u_{i}^{1} u_{j}^{2}\right\rangle$. To proceed, we note that for homogeneous and isotropic velocity fields, the correlation tensor $\left\langle u_{i}(\mathbf{0}) u_{j}(\mathbf{r})\right\rangle$ can be expressed as (Monin \& Yaglom 1975):

$$
\left\langle u_{i}(\mathbf{0}) u_{j}(\mathbf{r})\right\rangle=\mathscr{F}_{1} \hat{r}_{i} \hat{r}_{j}+\mathscr{F}_{2} \delta_{i j},
$$

where $\mathscr{F}_{1}$ and $\mathscr{F}_{2}$ are scalar functions of $r(r=|\mathbf{r}|)$ and $\hat{\mathbf{r}}$ is the unit vector in $\mathbf{r}$ direction. This implies, in particular, that $\left\langle u_{i}(\mathbf{0}) u_{j}(\mathbf{r})\right\rangle$ is symmetric in its indices $i$ and $j$, and therefore, $x_{i}^{1}\left\langle u_{i}^{1} u_{j}^{2}\right\rangle x_{j}^{2}=x_{i}^{1}\left\langle u_{i}^{2} u_{j}^{1}\right\rangle x_{j}^{2}$. Then from Eq. (3.7) and (3.9), we conclude that $T_{2}^{1}=T_{2}^{3}$, or in other words:

$$
\left\langle\overline{\mathbf{M}^{2}}\right\rangle=\left\langle\overline{\mathbf{M}}^{2}\right\rangle .
$$

Substituting in Eq. (3.6) leads to:

$$
\left\langle\overline{\mathbf{S}^{2}}\right\rangle=-\left\langle\overline{\mathbf{W}^{2}}\right\rangle+\frac{2}{3}\left\langle\overline{\mathbf{M}}^{2}\right\rangle
$$

Equations (3.11) and (3.12) can be viewed as generalizations of Eqs. (3.1) and (3.2) to the PVGT, for which $\overline{\mathbf{M}} \neq 0$. Obviously, Eqs. (3.11) and (3.12) reduce to the classical expressions when $R_{0}$ is in the dissipative range, where $\overline{\mathbf{M}}=0$.

\subsubsection{Expression of the second moments in terms of the two point structure functions}

For isotropic turbulent flows, the correlation functions $\left\langle u_{i}^{\alpha} u_{j}^{\beta}\right\rangle$ that appear in Eqs. (3.73.9) can in fact be systematically expressed in terms of the second order longitudinal velocity structure function

$$
D_{2}(r)=\left\langle[(\mathbf{U}(\mathbf{r})-\mathbf{U}(\mathbf{0})) \cdot \hat{\mathbf{r}}]^{2}\right\rangle
$$

where $\mathbf{U}$ is the fluctuating turbulent velocity. The velocity correlation tensor $\mathscr{R}_{i j}(\mathbf{r})=$ $\left\langle U_{i}(\mathbf{x}) U_{j}(\mathbf{x}+\mathbf{r})\right\rangle$ can be written as (Monin \& Yaglom 1975):

$$
\begin{aligned}
\mathscr{R}_{i j}(\mathbf{r}) & =\mathscr{R}_{1} \hat{r}_{i} \hat{r}_{j}+\mathscr{R}_{2} \delta_{i j} \\
& =\frac{\hat{r}_{i} \hat{r}_{j}}{4} r D_{2}^{\prime}(r)+\left[\frac{1}{3}\left\langle U^{2}\right\rangle-\frac{D_{2}(r)}{2}-\frac{r}{4} D_{2}^{\prime}(r)\right] \delta_{i j},
\end{aligned}
$$


where the prime, "'", denotes the derivative with respect to $r$. Recall the relation between $\mathbf{u}$ and $\mathbf{U}: \mathbf{u}^{\alpha}=\mathbf{U}^{\alpha}-\frac{1}{4}\left(\sum_{\beta=1}^{4} \mathbf{U}^{\beta}\right)$, and also that $\left\langle U_{i}^{1} U_{j}^{1}\right\rangle=\frac{1}{3}\left\langle U^{2}\right\rangle \delta_{i j},\left\langle U_{i}^{1} U_{j}^{2}\right\rangle=$ $\mathscr{R}_{i j}\left(\mathbf{x}^{2}-\mathbf{x}^{1}\right)$. The correlations appearing in Eqs. (3.7)-(3.9) can be expressed as:

$$
\begin{aligned}
\left\langle u_{i}^{1} u_{i}^{1}\right\rangle & =\left\langle\left(U_{i}^{1}-\frac{1}{4} \sum_{\beta=1}^{4} U_{i}^{\beta}\right)\left(U_{i}^{1}-\frac{1}{4} \sum_{\beta=1}^{4} U_{i}^{\beta}\right)\right\rangle \\
& =\left\langle U_{i}^{1} U_{i}^{1}\right\rangle-\frac{2}{4}\left(\left\langle U_{i}^{1} U_{i}^{1}\right\rangle+3\left\langle U_{i}^{1} U_{i}^{2}\right\rangle\right)+\frac{1}{16}\left(4\left\langle U_{i}^{1} U_{i}^{1}\right\rangle+12\left\langle U_{i}^{1} U_{i}^{2}\right\rangle\right) \\
& =\frac{3}{4}\left\langle U^{2}\right\rangle-\frac{3}{4}\left\langle U_{i}^{1} U_{i}^{2}\right\rangle=\frac{3}{4}\left\langle U^{2}\right\rangle-\frac{3}{4}\left(\mathscr{R}_{1}+3 \mathscr{R}_{2}\right) \\
& =\frac{9}{8} D_{2}\left(R_{0}\right)+\frac{3}{8} R_{0} D_{2}^{\prime}\left(R_{0}\right), \\
x_{i}^{1}\left\langle u_{i}^{1} u_{j}^{1}\right\rangle x_{j}^{1} & =x_{i}^{1}\left\langle\left(U_{i}^{1}-\frac{1}{4} \sum_{\beta=1}^{4} U_{i}^{\beta}\right)\left(U_{j}^{1}-\frac{1}{4} \sum_{\beta=1}^{4} U_{j}^{\beta}\right)\right\rangle x_{j}^{1} \\
& =\frac{3}{4} x_{i}^{1}\left\langle U_{i}^{1} U_{j}^{1}\right\rangle x_{j}^{1}-\frac{6}{4} x_{i}^{1}\left\langle U_{i}^{1} U_{j}^{2}\right\rangle x_{j}^{1}+\frac{6}{16} x_{i}^{1}\left\langle U_{i}^{1} U_{j}^{2}\right\rangle x_{j}^{1}+\frac{6}{16} x_{i}^{1}\left\langle U_{i}^{2} U_{j}^{3}\right\rangle x_{j}^{1} \\
& =\left(\frac{3}{32}\left\langle U^{2}\right\rangle-\frac{9}{32} \mathscr{R}_{1}-\frac{9}{32} \mathscr{R}_{2}\right) R_{0}^{2}=\frac{9}{64} D_{2}\left(R_{0}\right) R_{0}^{2},
\end{aligned}
$$

and

$$
\begin{aligned}
x_{i}^{1}\left\langle u_{j}^{1} u_{i}^{2}\right\rangle x_{j}^{2} & =x_{i}^{1}\left\langle\left(U_{i}^{1}-\frac{1}{4} \sum_{\beta=1}^{4} U_{i}^{\beta}\right)\left(U_{j}^{2}-\frac{1}{4} \sum_{\beta=1}^{4} U_{j}^{\beta}\right)\right\rangle x_{j}^{2} \\
& =\frac{1}{4} x_{i}^{1}\left\langle U_{i}^{1} U_{j}^{1}\right\rangle x_{j}^{2}+\frac{5}{8} x_{i}^{1}\left\langle U_{i}^{1} U_{j}^{2}\right\rangle x_{j}^{2}-\frac{8}{16} x_{i}^{1}\left\langle U_{i}^{1} U_{j}^{3}\right\rangle x_{j}^{2}+\frac{1}{8} x_{i}^{1}\left\langle U_{i}^{3} U_{j}^{4}\right\rangle x_{j}^{2} \\
& =\left(\frac{1}{96}\left\langle U^{2}\right\rangle-\frac{5}{32} \mathscr{R}_{1}-\frac{1}{32} \mathscr{R}_{2}\right) R_{0}^{2} \\
& =\left[\frac{1}{64} D_{2}\left(R_{0}\right)-\frac{1}{32} R_{0} D_{2}^{\prime}\left(R_{0}\right)\right] R_{0}^{2}
\end{aligned}
$$

where we have taken into account that the coordinates of the vertices $\mathbf{x}^{\alpha}$ can be expressed, up to a rotation, as $\left( \pm \frac{1}{2}, 0,-\frac{1}{2 \sqrt{2}}\right) R_{0}$ and $\left(0, \pm \frac{1}{2}, \frac{1}{2 \sqrt{2}}\right) R_{0}$. With the help of these expressions, Eq. (3.7)-(3.9) reduce to

$$
\left\langle\overline{\mathbf{M}}^{2}\right\rangle=\left\langle\overline{\mathbf{M}^{2}}\right\rangle=\frac{1}{R_{0}^{2}}\left(3 D_{2}\left(R_{0}\right)-\frac{3}{2} R_{0} D_{2}^{\prime}\left(R_{0}\right)\right),
$$

and

$$
\left\langle\overline{\mathbf{M M}^{\mathrm{T}}}\right\rangle=\frac{1}{R_{0}^{2}}\left(9 D_{2}\left(R_{0}\right)+3 r D_{2}^{\prime}\left(R_{0}\right)\right) .
$$

The expressions above can be further simplified by using the scaling properties of $D_{2}(r)$. In the inertial range of scales, $D_{2}(r)=C_{2}(\varepsilon r)^{2 / 3}$, so Eqs. (3.20), (3.19) together 
with Eq. (3.6), lead to

$$
\begin{aligned}
\left\langle\overline{\mathbf{M}}^{2}\right\rangle & =\left\langle\overline{\mathbf{M}^{2}}\right\rangle=2 \frac{D_{2}\left(R_{0}\right)}{R_{0}^{2}}, \\
\left\langle\overline{\mathbf{M M}^{\mathrm{T}}}\right\rangle & =11 \frac{D_{2}\left(R_{0}\right)}{R_{0}^{2}} \\
\left\langle\overline{\mathbf{S}^{2}}\right\rangle & =\frac{35}{6} \frac{D_{2}\left(R_{0}\right)}{R_{0}^{2}} \\
\left\langle\overline{\mathbf{W}^{2}}\right\rangle & =-\frac{9}{2} \frac{D_{2}\left(R_{0}\right)}{R_{0}^{2}}
\end{aligned}
$$

all for $\eta \ll R_{0} \ll L$. In the dissipative range of scales, $D_{2}(r)$ reduces to $D_{2}(r)=$ $\left\langle\left(m_{11}\right)^{2}\right\rangle r^{2}$, which yields

$$
\begin{aligned}
\left\langle\overline{\mathbf{M}}^{2}\right\rangle & =\left\langle\overline{\mathbf{M}^{2}}\right\rangle=0, \\
\left\langle\overline{\mathbf{M M}^{\mathrm{T}}}\right\rangle & =15 \frac{D_{2}\left(R_{0}\right)}{R_{0}^{2}}=15\left\langle\left(m_{11}\right)^{2}\right\rangle, \\
\left\langle\overline{\mathbf{S}^{2}}\right\rangle & =-\left\langle\overline{\mathbf{W}^{2}}\right\rangle=\frac{15}{2} \frac{D_{2}\left(R_{0}\right)}{R_{0}^{2}}=\frac{15}{2}\left\langle\left(m_{11}\right)^{2}\right\rangle,
\end{aligned}
$$

for $R_{0} \ll \eta$. As anticipated, one recovers in this limit the classical Betchov relations, Eqs. (3.1) and (3.2).

\subsection{Third-order invariants of $M$}

We now turn to the third-order invariants. As it was the case for the second-order moments, we introduce the notation $T_{3}^{p}$ for the third-order moments of $\mathbf{M}$ :

$$
T_{3}^{1}=\left\langle\overline{\mathbf{M}}^{3}\right\rangle, T_{3}^{2}=\left\langle\overline{\mathbf{M}^{2} \mathbf{M}^{\mathrm{T}}}\right\rangle, T_{3}^{3}=\left\langle\overline{\mathbf{M}^{2}} \overline{\mathbf{M}}\right\rangle, T_{3}^{4}=\left\langle\overline{\mathbf{M M}^{\mathrm{T}}} \overline{\mathbf{M}}\right\rangle \text {, and } T_{3}^{5}=\left\langle\overline{\mathbf{M}^{3}}\right\rangle .
$$

In homogeneous and isotropic turbulent flows, the corresponding quantities, when $R_{0}$ is in the dissipation range $\left(R_{0} \lesssim \eta\right)$, obtained by substituting $\mathbf{M}$ by $\mathbf{m}$ in the above definitions, all reduce to 0 , except for $T_{3}^{2}$. As we now show, the quantities $T_{3}^{1}, T_{3}^{3}$ and $T_{3}^{5}$ are in fact related through a simple relation.

To proceed, we express, as done in Section 3.1, the moments of the quantities $T_{3}^{p}$ in terms of moments of $x_{i}^{\alpha} x_{j}^{\beta} x_{k}^{\gamma}$, as well as the third order correlation velocity function, taken at two spatial points, $\mathscr{S}_{i j k}(\mathbf{r})=\left\langle U_{i}(\mathbf{x}) U_{j}(\mathbf{x}) U_{k}(\mathbf{x}+\mathbf{r})\right\rangle$ and the third order velocity correlation function evaluated at three different spatial points, forming an equilateral triangle: $\mathscr{Q}_{i j k}(\boldsymbol{\eta}, \boldsymbol{\xi})=\left\langle U_{i}(\mathbf{x}) U_{j}(\mathbf{x}+\boldsymbol{\eta}) U_{k}(\mathbf{x}+\boldsymbol{\xi})\right\rangle$. For incompressible isotropic fields, the third order correlation at two spatial points, $\mathscr{S}_{i j k}$, can be expressed as:

$$
\begin{aligned}
& \mathscr{S}_{i j k}(\mathbf{r})=\mathscr{S}_{1} \hat{r}_{i} \hat{r}_{j} \hat{r}_{k}+\mathscr{S}_{2}\left(\hat{r}_{i} \delta_{j k}+\hat{r}_{j} \delta_{i k}\right)+\mathscr{S}_{3} \hat{r}_{k} \delta_{i j} \\
& =\frac{1}{6}\left[\frac{D_{3}(r)-r D_{3}^{\prime}(r)}{2} \hat{r}_{i} \hat{r}_{j} \hat{r}_{k}+\frac{2 D_{3}(r)+r D_{3}^{\prime}(r)}{4}\left(\hat{r}_{i} \delta_{j k}+\hat{r}_{j} \delta_{i k}\right)-\frac{D_{3}(r)}{2} \hat{r}_{k} \delta_{i j}\right],
\end{aligned}
$$

where $D_{3}(r)=\left\langle[(\mathbf{U}(\mathbf{r})-\mathbf{U}(\mathbf{0})) \cdot \hat{\mathbf{r}}]^{3}\right\rangle$ is the third-order longitudinal velocity structure function. On the other hand, the three-point correlation function $\mathscr{Q}_{i j k}$ is not so well- 
known. It can be expressed as (Monin \& Yaglom 1975):

$$
\begin{aligned}
& \mathscr{Q}_{i j k}(\boldsymbol{\eta}, \boldsymbol{\xi})=\mathscr{Q}_{1} \hat{\eta}_{i} \hat{\eta}_{j} \hat{\eta}_{k}+\mathscr{Q}_{2} \hat{\eta}_{i} \delta_{j k}+\mathscr{Q}_{3} \hat{\eta}_{j} \delta_{i k} \\
& +\mathscr{Q}_{4} \hat{\eta}_{k} \delta_{i j}+\mathscr{Q}_{5} \hat{\eta}_{i} \hat{\eta}_{j} \hat{\xi}_{k}+\mathscr{Q}_{6} \hat{\eta}_{i} \hat{\xi}_{j} \hat{\eta}_{k}+\mathscr{Q}_{7} \hat{\xi}_{i} \hat{\eta}_{j} \hat{\eta}_{k} \\
& +\mathscr{Q}_{8} \hat{\xi}_{i} \hat{\xi}_{j} \hat{\eta}_{k}+\mathscr{Q}_{9} \hat{\xi}_{i} \hat{\eta}_{j} \hat{\xi}_{k}+\mathscr{Q}_{10} \hat{\eta}_{i} \hat{\xi}_{j} \hat{\xi}_{k}+\mathscr{Q}_{11} \hat{\xi}_{i} \hat{\xi}_{j} \hat{\xi}_{k} \\
& +\mathscr{Q}_{12} \hat{\xi}_{i} \delta_{j k}+\mathscr{Q}_{13} \hat{\xi}_{j} \delta_{i k}+\mathscr{Q}_{14} \hat{\xi}_{k} \delta_{i j},
\end{aligned}
$$

where $\mathscr{Q}_{n}$ 's are scalar functions of $|\boldsymbol{\xi}|,|\boldsymbol{\eta}|$, and $\boldsymbol{\xi} \cdot \boldsymbol{\eta}$. Using the symmetric conditions $\mathscr{Q}_{i j k}(\boldsymbol{\eta}, \boldsymbol{\xi})=\mathscr{Q}_{i k j}(\boldsymbol{\xi}, \boldsymbol{\eta})=\mathscr{Q}_{j i k}(-\boldsymbol{\eta}, \boldsymbol{\xi}-\boldsymbol{\eta})$, and noticing that for regular tetrahedron, $|\boldsymbol{\xi}|=|\boldsymbol{\eta}|=|\boldsymbol{\xi}-\boldsymbol{\eta}|=R_{0}$ and $\boldsymbol{\xi} \cdot \boldsymbol{\eta}=\boldsymbol{\xi} \cdot(\boldsymbol{\xi}-\boldsymbol{\eta})=\frac{1}{2} R_{0}^{2}$ (the three points involved in the definition of $\mathscr{Q}_{i j k}$ form an equilateral triangle), we can reduce the $\mathscr{Q}_{n}$ 's appearing in Eq. (3.26) to only three independent scalar functions:

$$
\begin{aligned}
& \mathscr{Q}_{i j k}(\boldsymbol{\eta}, \boldsymbol{\xi})=\mathscr{Q}_{1} \hat{\eta}_{i} \hat{\eta}_{j} \hat{\eta}_{k}+\mathscr{Q}_{2} \hat{\eta}_{i} \delta_{j k}-2 \mathscr{Q}_{2} \hat{\eta}_{j} \delta_{i k} \\
& +\mathscr{Q}_{2} \hat{\eta}_{k} \delta_{i j}+\mathscr{Q}_{5} \hat{\eta}_{i} \hat{\eta}_{j} \hat{\xi}_{k}-\frac{1}{2} \mathscr{Q}_{1} \hat{\eta}_{i} \hat{\xi}_{j} \hat{\eta}_{k}-\left(\mathscr{Q}_{1}+\mathscr{Q}_{5}\right) \hat{\xi}_{i} \hat{\eta}_{j} \hat{\eta}_{k} \\
& -\frac{1}{2} \mathscr{Q}_{1} \hat{\xi}_{i} \hat{\xi}_{j} \hat{\eta}_{k}+\mathscr{Q}_{5} \hat{\xi}_{i} \hat{\eta}_{j} \hat{\xi}_{k}-\left(\mathscr{Q}_{1}+\mathscr{Q}_{5}\right) \hat{\eta}_{i} \hat{\xi}_{j} \hat{\xi}_{k} \\
& +\mathscr{Q}_{1} \hat{\xi}_{i} \hat{\xi}_{j} \hat{\xi}_{k}+\mathscr{Q}_{2} \hat{\xi}_{i} \delta_{j k}+\mathscr{Q}_{2} \hat{\xi}_{j} \delta_{i k}-2 \mathscr{Q}_{2} \hat{\xi}_{k} \delta_{i j}
\end{aligned}
$$

Substituting Eqs. (3.24) and (3.27) into Eq. (3.23), a straightforward, although lengthy calculation leads to:

$$
\begin{aligned}
& T_{3}^{1}=\left(36 \mathscr{S}_{1}+36 \mathscr{S}_{2}+54 \mathscr{S}_{3}+72 \mathscr{Q}_{2}-48 \mathscr{Q}_{5}\right) / R_{0}^{3}, \\
& T_{3}^{2}=\left(30 \mathscr{S}_{1}+108 \mathscr{S}_{2}+42 \mathscr{S}_{3}+3 \mathscr{Q}_{1}-24 \mathscr{Q}_{2}+6 \mathscr{Q}_{5}\right) / R_{0}^{3}, \\
& T_{3}^{3}=\left(36 \mathscr{S}_{1}+60 \mathscr{S}_{2}+30 \mathscr{S}_{3}+24 \mathscr{Q}_{1}\right) / R_{0}^{3}, \\
& T_{3}^{4}=\left(30 \mathscr{S}_{1}+84 \mathscr{S}_{2}+66 \mathscr{S}_{3}-6 \mathscr{Q}_{1}+48 \mathscr{Q}_{2}-12 \mathscr{Q}_{5}\right) / R_{0}^{3}, \\
& T_{3}^{5}=\left(36 \mathscr{S}_{1}+72 \mathscr{S}_{2}+18 \mathscr{S}_{3}+36 \mathscr{Q}_{1}-36 \mathscr{Q}_{2}+24 \mathscr{Q}_{5}\right) / R_{0}^{3} .
\end{aligned}
$$

With these expressions, we notice that the quantities $T_{3}^{1}, T_{3}^{3}$ and $T_{3}^{5}$ are related by $\frac{1}{2} T_{3}^{1}-\frac{3}{2} T_{3}^{3}+T_{3}^{5}=0$, which can be re-expressed as:

$$
\left\langle\overline{\mathbf{M}^{3}}\right\rangle=\frac{3}{2}\left\langle\overline{\mathbf{M}^{2}} \overline{\mathbf{M}}\right\rangle-\frac{1}{2}\left\langle\overline{\mathbf{M}}^{3}\right\rangle
$$

Moreover, using the decomposition of $\mathbf{M}$, we relate the quantities $T_{3}^{p}$ to moments of $\mathbf{S}$, $\mathbf{W}$ and $\overline{\mathbf{M}}$ :

$$
\begin{aligned}
& T_{3}^{1}=\left\langle\overline{\mathbf{M}}^{3}\right\rangle, \\
& T_{3}^{2}=\left\langle\overline{\mathbf{S}^{3}}\right\rangle-\langle\overline{\mathbf{W S W}}\rangle+\left\langle\overline{\mathbf{S}^{2}} \overline{\mathbf{M}}\right\rangle-\frac{1}{3}\left\langle\overline{\mathbf{W}^{2}} \overline{\mathbf{M}}\right\rangle+\frac{1}{9}\left\langle\overline{\mathbf{M}}^{3}\right\rangle, \\
& T_{3}^{3}=\left\langle\overline{\mathbf{S}^{2}} \overline{\mathbf{M}}\right\rangle+\left\langle\overline{\mathbf{W}^{2}} \overline{\mathbf{M}}\right\rangle+\frac{1}{3}\left\langle\overline{\mathbf{M}}^{3}\right\rangle, \\
& T_{3}^{4}=\left\langle\overline{\mathbf{S}^{2}} \overline{\mathbf{M}}\right\rangle-\left\langle\overline{\mathbf{W}^{2}} \overline{\mathbf{M}}\right\rangle+\frac{1}{3}\left\langle\overline{\mathbf{M}}^{3}\right\rangle, \\
& T_{3}^{5}=\left\langle\overline{\mathbf{S}^{3}}\right\rangle+3\langle\overline{\mathbf{W S W}}\rangle+\left\langle\overline{\mathbf{S}^{2}} \overline{\mathbf{M}}\right\rangle+\left\langle\overline{\mathbf{W}^{2}} \overline{\mathbf{M}}\right\rangle+\frac{1}{9}\left\langle\overline{\mathbf{M}}^{3}\right\rangle,
\end{aligned}
$$

Substituting Eqs. (3.34), (3.34), and (3.34) into Eq. (3.29) yields:

$$
\left\langle\overline{\mathbf{S}^{3}}\right\rangle=-3\langle\overline{\mathbf{W S W}}\rangle+\frac{1}{2}\left\langle\overline{\mathbf{M}} \overline{\mathbf{M}^{2}}\right\rangle-\frac{5}{18}\left\langle\overline{\mathbf{M}}^{3}\right\rangle,
$$


Eq. (3.29) and (3.35) generalize the Betchov relations to the PVGT. They reduce to the classical expressions Eqs. (3.1) and (3.3) when $R_{0}$ in the dissipative range of scales $\left(R_{0} \lesssim \eta\right)$.

We note that, contrary to the second order invariants, for which we could explicitly express the invariants $T_{2}^{p}$, defined in Eq. (3.5), in terms of the well-studied second-order velocity structure function, the invariants $T_{3}^{p}$ cannot be reduced to the corresponding third-order structure function, expressed with two points only. Instead, they involve the three-point correlation functions based on 3-points forming an equilateral triangle, as shown by Eq. (3.28).

\section{Mixed second-order invariants of $M$ and $\Pi H$}

The evolution equations of the strain and enstrophy based on the PVGT, namely, Eqs. (2.18) and (2.19), involve not only the third-order invariants of $\mathbf{M}$ due to nonlinearity, but also the mixed invariants of $\mathbf{M}$ and $\mathbf{\Pi}$, which can be expressed in terms of $\langle\overline{\mathbf{M} \mathbf{H}}\rangle,\left\langle\overline{\mathbf{M}(\mathbf{\Pi H})^{\mathrm{T}}}\right\rangle$ and $\langle\overline{\mathbf{M}} \overline{\mathbf{\Pi H}}\rangle$. The terms involved in Eqs. (2.18) and (2.19) for the evolution of the strain and enstrophy, respectively $\langle\overline{\mathbf{\Pi H S}}\rangle$ and $\langle\overline{\mathbf{\Pi H} \mathbf{H}}\rangle$, can be readily deduced from these terms, via the relation:

$$
\langle\overline{\mathbf{\Pi H S}}\rangle=\frac{1}{2}\langle\overline{\mathbf{M} \mathbf{\Pi H}}\rangle+\frac{1}{2}\left\langle\overline{\mathbf{M}(\mathbf{\Pi H})^{\mathrm{T}}}\right\rangle-\frac{1}{3}\langle\overline{\mathbf{M}} \overline{\mathbf{\Pi H}}\rangle
$$

and

$$
\langle\overline{\mathbf{\Pi H W}}\rangle=\frac{1}{2}\langle\overline{\mathbf{M \Pi H}}\rangle-\frac{1}{2}\left\langle\overline{\mathbf{M}(\mathbf{\Pi H})^{\mathrm{T}}}\right\rangle .
$$

As we now demonstrate, these terms can also be represented by the third-order longitudinal structure function $D_{3}(r)$. We start by expanding these terms by their definitions to

$$
\begin{aligned}
\left\langle\overline{\mathbf{M}(\mathbf{\Pi H})^{\mathrm{T}}}\right\rangle & =\frac{8}{R_{0}^{2}}\left\langle u_{i}^{1} a_{i}^{1}\right\rangle, \\
\langle\overline{\mathbf{M}} \overline{\mathbf{\Pi}}\rangle & =\frac{4}{R_{0}^{4}}\left(4 x_{i}^{1}\left\langle x_{i}^{1} a_{j}^{1}\right\rangle x_{j}^{1}+12 x_{i}^{1}\left\langle u_{i}^{1} a_{j}^{2}\right\rangle x_{j}^{2}\right), \\
\langle\overline{\mathbf{M} \mathbf{\Pi}}\rangle & =\frac{4}{R_{0}^{4}}\left(4 x_{i}^{1}\left\langle u_{i}^{1} a_{j}^{1}\right\rangle x_{j}^{1}+12 x_{i}^{1}\left\langle a_{i}^{2} u_{j}^{1}\right\rangle x_{j}^{2}\right),
\end{aligned}
$$

in which the correlation between the reduced velocity $\mathbf{u}^{\alpha}$ and the reduced acceleration $\mathbf{a}^{\beta}$ can be related to the velocity-acceleration correlation function $\mathscr{L}_{i j}(\mathbf{r})=\left\langle U_{i}(\mathbf{x}) A_{j}(\mathbf{x}+\mathbf{r})\right\rangle$ through the definition $\mathbf{u}^{\alpha}=\mathbf{U}^{\alpha}-\frac{1}{4} \sum_{\beta=1}^{4} \mathbf{U}^{\beta}$ and $\mathbf{a}^{\alpha}=\mathbf{A}^{\alpha}-\frac{1}{4} \sum_{\beta=1}^{4} \mathbf{A}^{\beta}$. We also note that for isotropic flows, $\mathscr{L}_{i j}$ can be written as:

$$
\mathscr{L}_{i j}(\mathbf{r})=\mathscr{L}_{1} \hat{r}_{i} \hat{r}_{j}+\mathscr{L}_{2} \delta_{i j}
$$

in which $\mathscr{L}_{1}$ and $\mathscr{L}_{2}$ are scalar functions of $r$. With the help of Eq. (4.6), and using the symmetry of the vertices of the regular tetrads, we can evaluate the correlations involving $\left\langle u_{i}^{\alpha} a_{j}^{\beta}\right\rangle$ in terms of the velocity-acceleration correlation function as

$$
\begin{aligned}
\left\langle u_{i}^{1} a_{i}^{1}\right\rangle & =\frac{3}{4}\left\langle U_{i} A_{i}\right\rangle-\frac{3}{4}\left(\mathscr{L}_{1}+3 \mathscr{L}_{2}\right) \\
x_{i}^{1}\left\langle u_{i}^{1} a_{j}^{1}\right\rangle x_{j}^{1} & =\left(\frac{3}{32}\left\langle U_{i} A_{i}\right\rangle-\frac{9}{32} \mathscr{L}_{1}-\frac{9}{32} \mathscr{L}_{2}\right) R_{0}^{2} \\
x_{i}^{1}\left\langle u_{j}^{1} a_{i}^{2}\right\rangle x_{j}^{2} & =\left(\frac{1}{96}\left\langle U_{i} A_{i}\right\rangle-\frac{5}{32} \mathscr{L}_{1}-\frac{1}{32} \mathscr{L}_{2}\right) R_{0}^{2} .
\end{aligned}
$$


The stationarity of the flow implies that $\left\langle U_{i} A_{i}\right\rangle=\frac{d}{d t}\left\langle U^{2}\right\rangle=0$. We also note that the symmetry between the indices $i$ and $j$ leads to $\left\langle u_{i}^{1} a_{j}^{2}\right\rangle=\left\langle a_{i}^{2} u_{j}^{1}\right\rangle$.

To proceed, we need to derive tractable expressions for $\mathscr{L}_{1}$ and $\mathscr{L}_{2}$. To that end, we decompose the acceleration $\mathbf{A}$ as a sum of a local part $\partial \mathbf{U} / \partial t$, plus a convective part $\mathbf{U} \cdot \nabla \mathbf{U}$. This leads to:

$$
\begin{aligned}
\mathscr{L}_{i j}(\mathbf{r}) & =\left\langle U_{i}(\mathbf{x}) A_{j}(\mathbf{x}+\mathbf{r})\right\rangle \\
& =\left\langle U_{i}(\mathbf{x}) \frac{\partial U_{j}(\mathbf{x}+\mathbf{r})}{\partial t}\right\rangle+\left\langle U_{i}(\mathbf{x}) U_{k}(\mathbf{x}+\mathbf{r}) \frac{\partial U_{j}(\mathbf{x}+\mathbf{r})}{\partial(\mathbf{x}+\mathbf{r})_{k}}\right\rangle \\
& =\left\langle U_{i}(\mathbf{x}) \frac{\partial U_{j}(\mathbf{x}+\mathbf{r})}{\partial t}\right\rangle+\frac{\partial}{\partial r_{k}}\left\langle U_{i}(\mathbf{x}) U_{k}(\mathbf{x}+\mathbf{r}) U_{j}(\mathbf{x}+\mathbf{r})\right\rangle .
\end{aligned}
$$

The first term on the r.h.s. vanishes, since

$$
\begin{aligned}
0 & =\frac{\partial}{\partial t}\left\langle U_{i}(\mathbf{x}) U_{j}(\mathbf{x}+\mathbf{r})\right\rangle=\left\langle U_{i}(\mathbf{x}) \frac{\partial U_{j}(\mathbf{x}+\mathbf{r})}{\partial t}\right\rangle+\left\langle\frac{\partial U_{i}(\mathbf{x})}{\partial t} U_{j}(\mathbf{x}+\mathbf{r})\right\rangle \\
& =2\left\langle U_{i}(\mathbf{x}) \frac{\partial U_{j}(\mathbf{x}+\mathbf{r})}{\partial t}\right\rangle,
\end{aligned}
$$

where the last equality comes from isotropy. Finally, since by definition $\left\langle U_{i}(\mathbf{x}) U_{k}(\mathbf{x}+\mathbf{r}) U_{j}(\mathbf{x}+\mathbf{r})\right\rangle=$ $\mathscr{S}_{j k i}(-\mathbf{r})$, we obtain from Eq. (4.10),

$$
\mathscr{L}_{i j}(\mathbf{r})=\frac{\partial}{\partial r_{k}} S_{j k i}(-\mathbf{r})
$$

Straightforward manipulations lead to:

$$
\begin{aligned}
\mathscr{L}_{i j}(\mathbf{r}) & =\mathscr{L}_{1} \hat{r}_{i} \hat{r}_{j}+\mathscr{L}_{2} \delta_{i j} \\
& =\left(\frac{-2 \mathscr{S}_{1}}{r}-\mathscr{S}_{1}^{\prime}+\frac{\mathscr{S}_{2}}{r}-\mathscr{S}_{2}^{\prime}+\frac{\mathscr{S}_{3}}{r}-\mathscr{S}_{3}^{\prime}\right) \hat{r}_{i} \hat{r}_{j}+\left(\frac{-3 \mathscr{S}_{2}}{r}-\mathscr{S}_{2}^{\prime}-\frac{\mathscr{S}_{3}}{r}\right) \delta_{i j} \\
& =\left(\frac{-D_{3}(r)}{6 r}+\frac{D_{3}^{\prime}(r)}{6}+\frac{D_{3}^{\prime \prime}(r) r}{24}\right) \hat{r}_{i} \hat{r}_{j}+\left(\frac{-D_{3}(r)}{6 r}-\frac{D_{3}^{\prime}(r)}{4}-\frac{D_{3}^{\prime \prime}(r) r}{24}\right) \delta_{i j} .
\end{aligned}
$$

Therefore, substituting Eqs. (4.7)-(4.9) and (4.13) into Eqs. (4.3)-(4.5), we obtain

$$
\begin{aligned}
\left\langle\overline{\mathbf{M}(\mathbf{\Pi H})^{\mathrm{T}}}\right\rangle & =\frac{4 D_{3}\left(R_{0}\right)}{R_{0}}+\frac{7 D_{3}^{\prime}\left(R_{0}\right)}{2}+\frac{D_{3}^{\prime \prime}\left(R_{0}\right) R_{0}}{2}, \\
\langle\overline{\mathbf{M} \Pi \mathbf{H}}\rangle & =\langle\overline{\mathbf{M}} \overline{\mathbf{\Pi}}\rangle=\frac{3 D_{3}\left(R_{0}\right)}{R_{0}}-\frac{D_{3}^{\prime}\left(R_{0}\right)}{2}-\frac{D_{3}^{\prime \prime}\left(R_{0}\right) R_{0}}{4} .
\end{aligned}
$$

Eqs. (4.14) and (4.15) can be further simplified by using the scaling properties of the structure function $D_{3}(r)$. For $R_{0}$ in the inertial range of scales, the celebrated four-fifths law, $D_{3}\left(R_{0}\right)=-\frac{4}{5} \varepsilon R_{0}$, implies

$$
\left\langle\overline{\mathbf{M}(\mathbf{\Pi H})^{\mathrm{T}}}\right\rangle=\frac{15 D_{3}\left(R_{0}\right)}{2 R_{0}^{3}}=\frac{-6 \varepsilon}{R_{0}^{2}}
$$

and

$$
\langle\overline{\mathbf{M \Pi H}}\rangle=\langle\overline{\mathbf{M}} \overline{\mathbf{\Pi}} \overline{\mathbf{H}}\rangle=\frac{5 D_{3}\left(R_{0}\right)}{2 R_{0}^{3}}=\frac{-2 \varepsilon}{R_{0}^{2}}
$$


When $R_{0}$ is in the dissipative range, $D_{3}\left(R_{0}\right)=\left\langle\left(m_{11}\right)^{3}\right\rangle R_{0}^{3}$, which leads to

$$
\left\langle\overline{\mathbf{M}(\mathbf{\Pi H})^{\mathrm{T}}}\right\rangle=\frac{35 D_{3}\left(R_{0}\right)}{2 R_{0}^{3}}=\frac{35}{2}\left\langle\left(m_{11}\right)^{3}\right\rangle
$$

and

$$
\langle\overline{\mathbf{M} \Pi \mathbf{H}}\rangle=\langle\overline{\mathbf{M}} \overline{\mathbf{\Pi H}}\rangle=0 .
$$

It is interesting to recall that the acceleration $\mathbf{A}^{\alpha}$ can be decomposed as a sum of the pressure gradient, viscous dissipation and external forcing, see Eq. (2.7). The mixed second-order invariants of $\mathbf{M}$ and $\mathbf{\Pi H}$ considered in this section can be divided into three parts, corresponding to contribution from the external forcing term, the pressure gradient term and the viscous term. We expect the viscous term to dominate the other two, especially when the length scale is much smaller than the integral scale $L$. The arguments are that first, $\left\langle U(\mathbf{x})_{i} \nabla_{j} P(\mathbf{x}+\mathbf{r})\right\rangle=0$ due to isotropy and incompressibility (von Kármán $\&$ Howarth 1938) and second, the external force $\mathbf{F}^{\alpha}$ is imposed on the large scale and varies moderately among the region we consider, thus $\mathbf{f}^{\alpha}=\mathbf{F}^{\alpha}-\frac{1}{4} \sum_{\beta=1}^{4} \mathbf{F}^{\beta} \approx 0$, which leads to $\left\langle u_{i}^{\alpha} f_{j}^{\beta}\right\rangle \approx 0$.

\section{Verification of the theoretical predictions: DNS results}

In this section we examine the theoretical results derived in Sections 2, 3 and 4, using DNS data. In addition to checking our derivation, numerical data provides useful information on $T_{3}^{p}$, the third moments of $\mathbf{M}$ (see Eq. (3.23)), which depend on a largely undocumented three-point velocity correlation function, see Section 3.2.

Three different data sets with Reynolds number $R_{\lambda}=166,406$, and 610 are used. The $R_{\lambda}=166$ data set was generated by using a spectral code, run on the cluster at ENS Lyon with a $384^{3}$ spatial resolution. The other two sets were downloaded from the Johns Hopkins University database( $\mathrm{Li}$ et al. 2008; Yeung et al. 2012). In order to construct the PVGT from regular tetrahedra with various sizes, we notice that four points out of the eight vertices of a cube form a regular tetrahedron if every two of them are on a surface diagonal line, which provides a convenient approach to extract data points forming tetrahedra from a regular cubic grid out of the simulation domain. The smallest tetrad size $R_{0, \text { min }}$ that can be reached is then $\sqrt{2}$ times the grid spacing. Tetrads with sizes in integer numbers of $R_{0, \text { min }}$ can also be obtained without interpolation. The eight vertices of a cube form two tetrahedra with different orientations, and for each orientation the number of statistics equals to the number of spatial points taking into account of the periodical boundary conditions of the simulation. For data set $R_{\lambda}=166$ we extract $384^{3}$ data points from two different snapshots, results in statistics of $2 \times 384^{3} \approx 1.1 \times 10^{8}$ for each orientation and $R_{0, \min } / \eta \approx 2.8$. For the other two Reynolds numbers, we extract $512^{3}$ data points from one single snapshot, which allows us to obtain statistics with $512^{3} \approx 1.3 \times 10^{8}$ data points for each orientation and $R_{0, \min } / \eta \approx 6.3$ at $R_{\lambda}=406$ and $R_{0, \min } / \eta \approx 12.5$ at $R_{\lambda}=610$. To check the statistical convergence, we compared the values obtained from two different tetrahedron orientations, and in all cases they differ by no more than a few percents.

Figure 1(a) and (b) verify the generalized Betchov relations, Eq. (3.12) and (3.35) respectively. Namely, the left-hand (square symbols) and right-hand (cross symbols) sides of Eqs. (3.12) and (3.35), made dimensionless by using the Kolmogorov time scale corresponding to the tetrad size, $t_{0} \equiv\left(R_{0}^{2} / \varepsilon\right)^{1 / 3}$, are plotted. For Eq. (3.35), we actually plotted the negative values of both sides as $\left\langle\overline{\mathbf{S}^{3}}\right\rangle<0$. Our results show that the two 


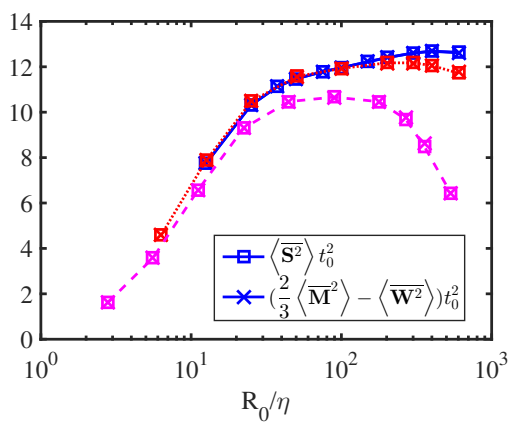

(a)

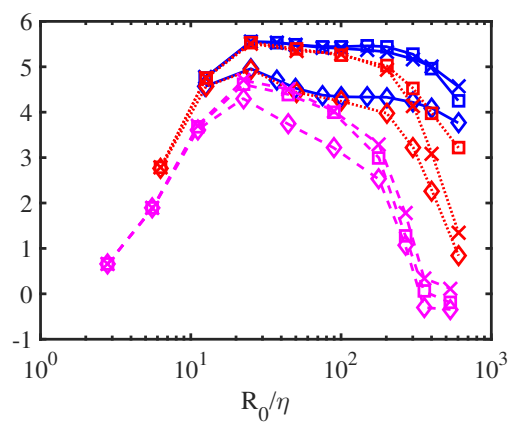

(b)

Figure 1. Generalized Betchov relations Eq. (3.12) and (3.35). (a): 1.h.s. (squares) and r.h.s. (crosses) of Eq. (3.12); (b): negative values of the l.h.s. (squares) and r.h.s. (crosses) of Eq. (3.35). The values of $3\langle\overline{\mathbf{W S W}}\rangle$ (diamonds) are also shown in (b) for comparison. All terms are made dimensionless by using the time scale $t_{0} \equiv\left(R_{0}^{2} / \varepsilon\right)^{1 / 3}$. In both panels, the results obtained at Reynolds numbers $R_{\lambda}=610,406$ and 166, are shown by the solid blue line, the red dotted and the magenta dashed lines, respectively.

sets of symbols superpose almost perfectly for both second and third-order quantities. The imbalance between the two sides of the equation, clearly visible at large values of $R_{0} / \eta$ in the case of the third-order moments is very likely due to the residual large-scale anisotropy, since the equations have been derived under the explicit assumption of homogeneity and isotropy of the flow. Moreover, we notice that, when normalized by $t_{0}$, the results at the two higher Reynolds numbers, $R_{\lambda}=406$ and 610 collapse well for $R_{0}$ smaller than the integral length scale $L$. This is an indication that the properties of the inertial range dynamics studied here with the PVGT are indeed universal for highReynolds number turbulence. The values at $R_{\lambda}=166$ are in general lower than those for the higher Reynolds number runs, an effect that we attribute to the finite Reynolds number effect.

In Fig. 1(b) is also shown the values of the term corresponding to vortex stretching, $3\langle\overline{\mathbf{W S W}}\rangle$, shown with diamond symbols. One can see that in the dissipative range, this quantity is identical to $-\left\langle\overline{\mathbf{S}^{3}}\right\rangle$ as implied by Eq. (3.3). At larger values of $R_{0}, 3\langle\overline{\mathbf{W S W}}\rangle$ is only slightly smaller than $-\left\langle\overline{\mathbf{S}^{3}}\right\rangle$ (we will return to this ratio, see Fig. 2d). This indicates that the relation between the third moment of strain and vortex stretching, established in Eq. (3.3), provides a good approximation even in the inertial range.

Further insight on the generalized Betchov relations can be obtained by comparing the various terms in Eqs. (3.12) and (3.35). Figure 2(a) shows the terms in Eq. (3.12), all made dimensionless by dividing by $D_{2}\left(R_{0}\right) / R_{0}^{2}$. The horizontal lines correspond to the exact values in the dissipative or the inertial range, as predicted by the calculations in Section 3.1.2, see Eqs.(3.19-3.21). For values of $R_{0} \lesssim 4 \eta$, the values of $\left\langle\overline{\mathbf{M}^{2}}\right\rangle,\left\langle\overline{\mathbf{S}^{2}}\right\rangle$, and $\left\langle\overline{\mathbf{W}^{2}}\right\rangle$ agree with the asymptotic limit predicted in the dissipative range. Similarly, for $R_{0} \gtrsim 50 \eta$, these quantities follow the predicted behavior in the inertial range. The small value of $\left\langle\overline{\mathbf{M}^{2}}\right\rangle$, compared to either of $\left\langle\overline{\mathbf{S}^{2}}\right\rangle$ or $\left\langle\overline{\mathbf{W}^{2}}\right\rangle$, ensures that the ratio $-\left\langle\overline{\mathbf{S}^{2}}\right\rangle /\left\langle\overline{\mathbf{W}^{2}}\right\rangle$ does not deviate by more than $\sim 30 \%$ with respect to 1 . As shown in Fig. $2 \mathrm{~b}$, the ratio $-\left\langle\overline{\mathbf{S}^{2}}\right\rangle /\left\langle\overline{\mathbf{W}^{2}}\right\rangle$ increases monotonically from 1 in in the dissipative range to the predicted value of $\frac{35}{27}$ when $R_{0}$ increases in the inertial range. We stress that at the level of the PVGT, for $R_{0}$ above the dissipative range, or $R_{0} \gtrsim 10 \eta$, strain dominates over enstrophy.

The values of $\left\langle\overline{\mathbf{M}}^{3}\right\rangle$ and $\left\langle\overline{\mathbf{M}} \overline{\mathbf{M}}^{2}\right\rangle$, made dimensionless by dividing by $D_{3}\left(R_{0}\right) / R_{0}^{3}$, are 


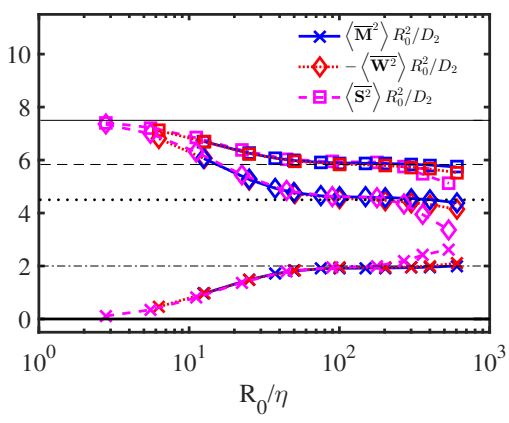

(a)

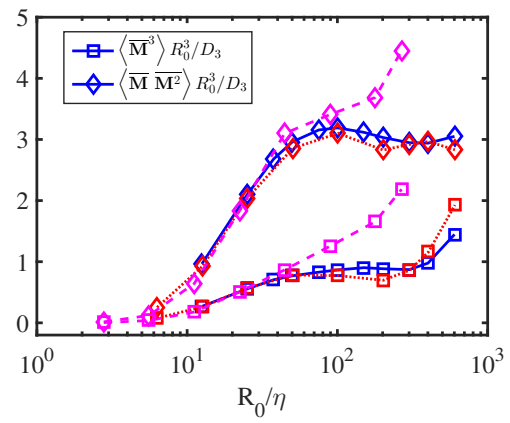

(c)

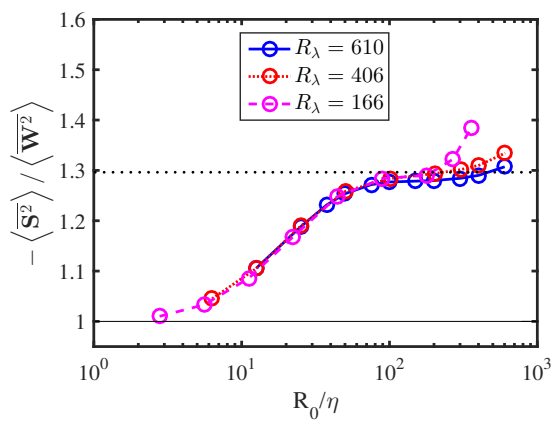

(b)

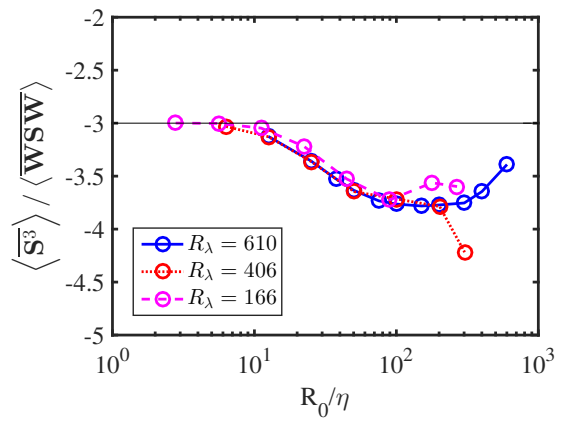

(d)

FIGURE 2. DNS results for the second- and third-order invariants of $\mathbf{M}$. In all subfigures, blue solid, red dotted and magenta dashed curves indicate Reynolds numbers $R_{\lambda}=610,406$ and 166, respectively. (a) Values of $\left\langle\overline{\mathbf{M}}^{2}\right\rangle$ (crosses), $-\left\langle\overline{\mathbf{W}^{2}}\right\rangle$ (diamonds) and $\left\langle\overline{\mathbf{S}^{2}}\right\rangle$ (squares), all normalized by $D_{2}\left(R_{0}\right) / R_{0}^{2}$, at different scale $R_{0} / \eta$. The straight lines show the theoretical predictions: $\left\langle\overline{\mathbf{S}^{2}}\right\rangle=-\left\langle\overline{\mathbf{W}^{2}}\right\rangle=\frac{15}{2}$ (the thin solid line) and $\left\langle\overline{\mathbf{M}}^{2}\right\rangle=0$ (thick solid line) in the dissipative range, and $\left\langle\overline{\mathbf{S}^{2}}\right\rangle=\frac{35}{6}$ (thin dashed line), $-\left\langle\overline{\mathbf{W}^{2}}\right\rangle=\frac{9}{2}$ (thin dotted line) and $\left\langle\overline{\mathbf{M}}^{2}\right\rangle=2$, (thin dot-dashed line) in the inertial range. (b) Change of the ratio $-\left\langle\overline{\mathbf{S}^{2}}\right\rangle /\left\langle\overline{\mathbf{W}^{2}}\right\rangle$ with $R_{0} / \eta$. The thin solid and the dotted lines show the predicted values of 1 and $\frac{35}{27}$ in the dissipative and inertial ranges. (c) Values of $\left\langle\overline{\mathbf{M}}^{3}\right\rangle$ (squares) and $\left\langle\overline{\mathbf{M}} \overline{\mathbf{M}^{2}}\right\rangle$ (diamonds), normalized by $D_{3}\left(R_{0}\right) / R_{0}^{3}$, at different scale $R_{0} / \eta$. (d) Dependence of the ratio $\left\langle\overline{\mathbf{S}^{3}}\right\rangle /\langle\overline{\mathbf{W S W}}\rangle$ as a function of scale $R_{0} / \eta$. The solid line is the theoretical value -3 in the dissipative range.

shown in Fig. $(2)(\mathrm{c})$. Note that $D_{3}\left(R_{0}\right)<0$, so the positive values in the Figure imply that the two quantities shown are in fact negative. Whereas in the dissipative range, for $R_{0} \lesssim 4 \eta$, all the quantities are very close to 0 , both $\left\langle\overline{\mathbf{M}}^{3}\right\rangle$ and $\left\langle\overline{\mathbf{M}} \overline{\mathbf{M}}^{2}\right\rangle$ approximately show a plateau when $R_{\lambda} \gtrsim 400$. This is not surprising, since the correlation functions $\mathscr{Q}_{i}$ 's in Eqs. (3.28) are expected to scale with the same exponent as $D_{3}$ in the inertial range. In the same spirit, the ratio between $\left\langle\overline{\mathbf{S}^{3}}\right\rangle$ and $\langle\overline{\mathbf{W S W}}\rangle$ decreases from the theoretical prediction -3 in the dissipative range to another constant value, approximately equal to -3.7 in the inertial range. Although this ratio determined numerically in the inertial range differs slightly from that predicted by the original Betchov relation in the dissipative range, Eq. (3.3), the qualitative picture is unchanged: the positive value of (perceived) vortex stretching $\langle\overline{\mathbf{W S W}}\rangle>0$, corresponds to $\left\langle\overline{\mathbf{S}^{3}}\right\rangle<0$.

Figure 3 presents the values of the mixed second-order invariants of $\mathbf{M}$ and $\mathbf{\Pi H}$, 


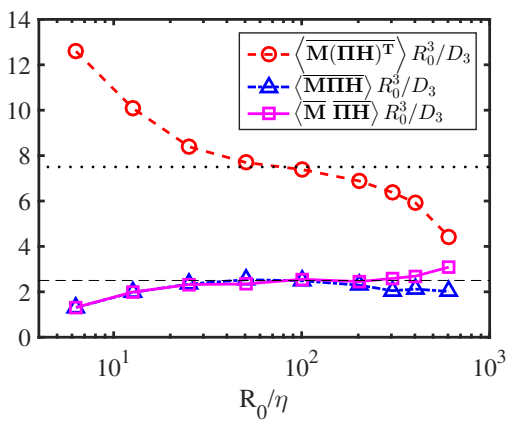

(a)

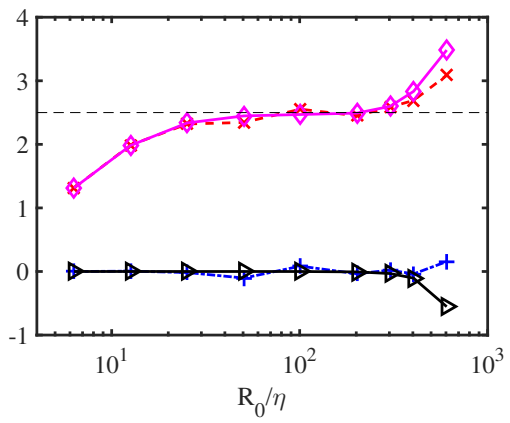

(c)

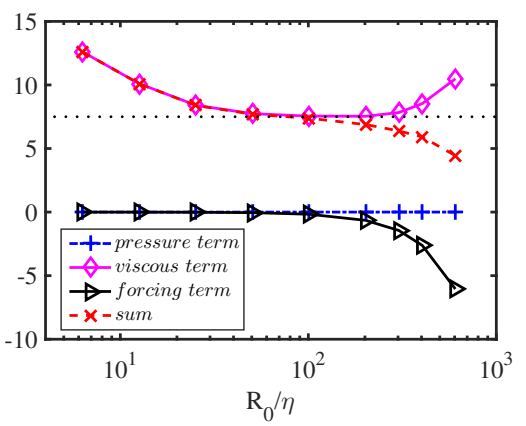

(b)

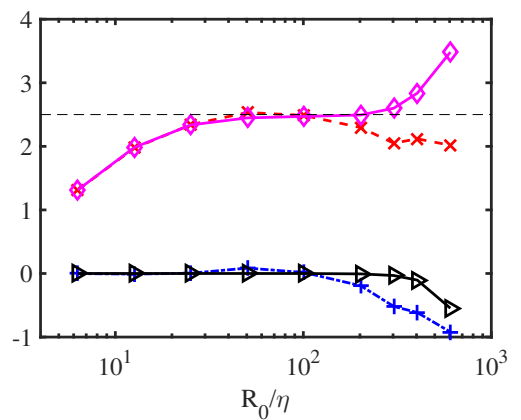

(d)

FIgURE 3. DNS results for the mixed second-order invariants of $\mathbf{M}$ and $\mathbf{\Pi H}$ at $R_{\lambda}=406$. (a) $\left\langle\overline{\mathbf{M}(\mathbf{\Pi H})^{\mathrm{T}}}\right\rangle$ (crosses), $\langle\overline{\mathbf{M} \boldsymbol{\Pi} \mathbf{H}}\rangle$ (pluses) and $\langle\overline{\mathbf{M}} \overline{\mathbf{\Pi H}}\rangle$ (diamonds) normalized by $D_{3}\left(R_{0}\right) / R_{0}^{3}$. (b) Contributions of the pressure gradient term (blue pluses), the viscous term (magenta diamonds) and the external forcing (black triangles) to $\left\langle\overline{\mathbf{M}(\mathbf{\Pi H})^{\mathrm{T}}}\right\rangle$ and their sum (red crosses), all normalized by $D_{3}\left(R_{0}\right) / R_{0}^{3}$. (c) Same as (b), but for $\langle\overline{\mathbf{M} \Pi \mathbf{H}}\rangle$. (d) Same as (b), but for $\langle\overline{\mathbf{M}} \overline{\mathbf{\Pi}} \mathbf{H}\rangle$. The straight lines are theoretical predictions in the inertial range for $\left\langle\overline{\mathbf{M}(\mathbf{\Pi H})^{\mathrm{T}}}\right\rangle\left(=\frac{15}{2}\right.$, dotted line) and $\langle\overline{\mathbf{M} \Pi \mathbf{H}}\rangle=\langle\overline{\mathbf{M}} \overline{\mathbf{\Pi}}\rangle\left(=\frac{5}{2}\right.$, dashed line $)$.

determined only from the $R_{\lambda}=406$ flow. (The external forcing terms in the $R_{\lambda}=166$ and $R_{\lambda}=610$ cases are not available so making the analysis of the $\mathbf{\Pi H}$ term impossible in these two cases.) The smallest value of $R_{0} \approx 6.3 \eta$ does not allow us to explore directly the dissipative range. On the other hand, our results is compatible with the existence of a plateau in the inertial range of scales for $\langle\overline{\mathbf{M} \mathbf{H}}\rangle$ and for $\langle\overline{\mathbf{M}} \overline{\mathbf{\Pi}} \overline{\mathbf{H}}\rangle$, consistent with

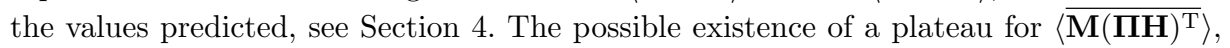
however, is at best suggested by the inflection point in Fig. 3.

In the inertial range, the theoretical predictions give $\left\langle\overline{\mathbf{M}(\mathbf{\Pi H})^{\mathrm{T}}}\right\rangle-\langle\overline{\mathbf{M \Pi H}}\rangle \approx(15 / 2-$ $5 / 2) \times-\frac{4}{5} \varepsilon \approx-4 \varepsilon<0$, which is consistent with the numerical results. This leads to the conclusion that the generation of perceived enstropy is positive, which is plausible considering the picture of turbulence cascade, $\langle\overline{\mathbf{W S W}}\rangle>0$. As a consequence, $-\left\langle\overline{\mathbf{S}^{3}}\right\rangle$ should be greater than 0 , which says that the intermediate eigenvalue is preferentially positive.

In subfigures (b), (c) and (d) of Fig. 3, we decompose the values of those mixed invariants into three parts, corresponding to the force, pressure and viscous terms. Consistent 


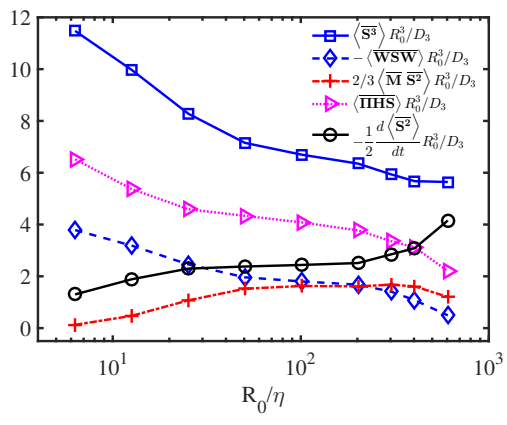

(a)

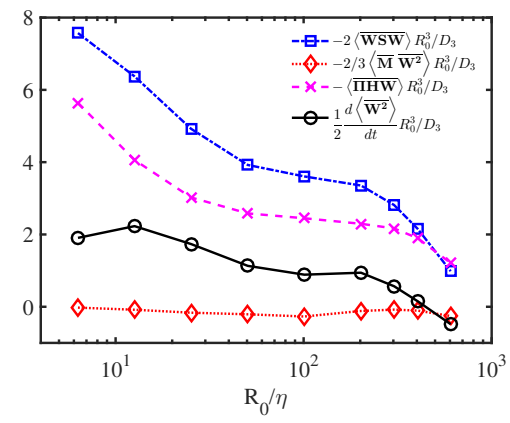

(b)

FiguRE 4. Magnitudes of terms in the equation for the rate of production of strain, Eq. (2.18) (a) and vorticity, Eq. (2.19) (b). All terms are made dimensionless by dividing by $D_{3}\left(R_{0}\right) / R_{0}^{3}$ (note that $D_{3}\left(R_{0}\right)<0$ ).

with our previous analysis, when $R_{0} \ll L$, the contribution from the external force and the pressure gradient terms are negligible. The situation changes when $R_{0} \simeq L$, which is likely to be a consequence of the flow anisotropy at scales comparable to the size of the simulation domain.

Finally, Fig. 4 shows the terms in the equation for the rate of production of strain and vorticity obtained from PVGT based on regular tetrads, i.e., Eq. (2.18) and Eq. (2.19). Note that the rates of production for quantities from PVGT are non-zero even in statistically stationary turbulence because the tetrads evolve in size and shape as the fluid particles forming the tetrads move in the flow. The data shown in Fig. 4 is from DNS at $R_{\lambda}=406$. All the terms in these two equations are made dimensionless by dividing by $D_{3}\left(R_{0}\right) / R_{0}^{3}$. We multiplied the various contributions by \pm 1 to make all the quantities positive (note that $D_{3}\left(R_{0}\right)$ is negative). As an example, we plot $\left\langle\overline{\mathbf{S}^{3}}\right\rangle R_{0}^{3} / D_{3}\left(R_{0}\right)$ instead of $-\left\langle\overline{\mathbf{S}^{3}}\right\rangle R_{0}^{3} / D_{3}\left(R_{0}\right)$ that appears in Eq. (2.18). Note that since $\left\langle\overline{\mathbf{S}^{2}}\right\rangle>0$ and $\left\langle\overline{\mathbf{W}^{2}}\right\rangle<0$, the DNS data shown in Fig. 4 indicate that $\frac{d}{d t}\left\langle\overline{\mathbf{S}^{2}}\right\rangle>0$ and $\frac{d}{d t}\left\langle\overline{\mathbf{W}^{2}}\right\rangle<0$, i.e., the magnitudes of both strain and vorticity are increasing. From Fig. $4(\mathrm{a})$, we see that the largest contribution to the production of strain comes from $-\left\langle\overline{\mathbf{S}^{3}}\right\rangle$, with additional small positive contribution from the term $-\frac{2}{3}\left\langle\overline{\mathbf{M}} \overline{\mathbf{S}^{2}}\right\rangle$. The term $\langle\overline{\mathbf{M H S}}\rangle$, on the other hand, acts against the production of strain. This contribution describes the action of other forces against the deformation of the tetrads, and, as we noticed when discussing Fig. 3, is mostly due to the viscous dissipation. Expressing $\langle\overline{\mathbf{\Pi H S}}\rangle \approx\left(\langle\overline{\mathbf{\Pi H M}}\rangle+\left\langle\overline{\mathbf{\Pi H} \mathbf{M}^{\mathrm{T}}}\right\rangle\right) / 2$, and using Eqs. $(4.14,4.15)$ leads to the prediction that $\langle\overline{\mathbf{I H S}}\rangle \times R_{0}^{3} / D_{3}=\left(\frac{5}{2}+\frac{15}{2}\right) / 2=5$ in the inertial range, which is well supported by data shown in Fig. 4(a). In addition, the vortex stretching term, $\langle\overline{\mathbf{W S W}}\rangle$, also provides a negative contribution to the rate of change of $\left\langle\overline{\mathbf{S}^{2}}\right\rangle$.

In the case of the rate of production of enstrophy, Fig. 4(b) shows that the main positive contribution is the vortex stretching term $\langle\overline{\mathbf{W S W}}\rangle$, as expected. The term originating from the non-zero value of $\overline{\mathbf{M}},-\frac{2}{3}\left\langle\overline{\mathbf{M}} \overline{\mathbf{W}^{2}}\right\rangle$, is negligibly small over the entire range of $R_{0}$ explored. The forcing term $\langle\overline{\mathbf{\Pi H W}}\rangle$ contributes negatively to the production of enstrophy. Using the identity $\langle\overline{\mathbf{\Pi H W}}\rangle \approx\left(\langle\overline{\mathbf{\Pi H M}}\rangle-\left\langle\overline{\mathbf{\Pi} \mathbf{H ~ M}^{\mathrm{T}}}\right\rangle\right) / 2$, as well as Eqs. (4.14) and (4.15) when $R_{0}$ is in the inertial range of scales, one obtains: $-\langle\overline{\mathbf{\Pi H W}}\rangle R_{0}^{3} / D_{3} \approx$ $-\left(\frac{5}{2}-\frac{15}{2}\right) / 2=\frac{5}{2}$, which is also well supported by the data shown in Fig. 4(b).

As the terms in the equation for the rate of production of strain and vorticity, $\frac{1}{2} \frac{d}{d t}\left\langle\overline{\mathbf{S}^{2}}\right\rangle$ 
and $\frac{1}{2} \frac{d}{d t}\left\langle\overline{\mathbf{W}^{2}}\right\rangle$, are shown in the two panels of Fig. 4 with the same normalization, they could thus be compared directly. We notice that, except in the dissipative range when $R_{0} \lesssim \eta$, strain production is much larger than enstrophy production, approximately by a factor of 3 in the inertial range (Note that the vertical range of the two panels are different.) As already noticed, the evolution of strain and enstrophy measured with regular tetrads of size $R_{0}$, does not give rise to a closed, stationary problem, as the shape and size of the tetrads evolve with time, an effect that has to be taken into account in a consistent description of the problem (Pumir et al. 2013). The excess of strain production, compared to that of vorticity production is nonetheless consistent with the excess of strain over enstrophy when $R_{0}$ is in the inertial range, as clearly seen in our prediction Eq. (3.21) and from data shown in Fig. 2(b). Thus, our results at a finite scale, with $R_{0}$ in the inertial range, consistently point to an excess of strain, compared to enstrophy.

\section{Discussion and concluding remarks}

In this article, we have established exact equations for the evolution of the perceived velocity gradient tensor, M, constructed from 4 points in the fluid, forming a regular tetrad of size $R_{0}$. Whereas the dynamics of $\mathbf{M}$ shares many similarities with that of the true velocity gradient tensor, $\mathbf{m}$, an important difference comes from the trace of $\mathbf{M}$, which is not identically 0 . The usual decomposition of $\mathbf{M}$ in terms of its symmetric, $\mathbf{S}$, and antisymmetric, $\mathbf{W}$ components, has to be generalized to take into account the non-zero $\overline{\mathbf{M}} \equiv \operatorname{tr}(\mathbf{M})$, see Eq. (2.5).

Starting from the incompressible N-S equations, we derived the evolution equation of the rate of strain and enstrophy of the PVGT. We also extended the well-known Betchov relations, originally for $\mathbf{m}$, to $\mathbf{M}$, to relate the norms of strain $\left\langle\overline{\mathbf{S}^{2}}\right\rangle$ and enstrophy $-\left\langle\overline{\mathbf{W}^{2}}\right\rangle$ in the flow, as well as vortex stretching $\langle\overline{\mathbf{W S W}}\rangle$ and the third moment of strain, $\left\langle\overline{\mathbf{S}^{3}}\right\rangle$.

In addition, in a homogeneous and isotropic turbulent flow, we expressed all the second order invariants of $\mathbf{M}$ in terms of the well-documented second-order structure function, $D_{2}\left(R_{0}\right)$. On the other hand, an exact expression of the third-order invariants of $\mathbf{M}$ require, in addition to the structure function $D_{3}\left(R_{0}\right)$, a correlation function of the velocity field involving 3 points on an equilateral triangle.

Using DNS data of homogeneous and isotropic turbulence, we find that when $R_{0}$ is in the inertial range, the ratio $-\left\langle\overline{\mathbf{S}^{2}}\right\rangle /\left\langle\overline{\mathbf{W}^{2}}\right\rangle$ is approximately 1.3 (compared to 1 in the dissipative range), in excellent agreement with our theoretical value of $\frac{35}{27}$. We also demonstrate numerically that, in the inertial range of scales, the production of strain significantly exceeds that of enstrophy. These results, taken together, suggest the prevalence of strain over vorticity at the level of $\mathbf{M}$, an effect anticipated several times (Tsinober 2009; Carbone \& Bragg 2020). The description in terms of the perceived velocity gradient tensor may therefore lead to insight previously difficult to obtain, at the inertial range of scales.

\section{Acknowledgements}

We acknowledge financial support from the National Science Foundation of China (NSFC) under grant number 11672157. AP received support from the IDEXLyon project (Contract No. ANR-16-IDEX-0005) under University of Lyon auspices. We are very thankful to A. Bragg for many fruitful discussions. 


\section{Declaration of Interest}

The authors report no conflict of interest.

\section{REFERENCES}

Betchov, R. 1956 An inequality concerning the production of vorticity in isotropic turbulence. J. Fluid Mech. 1, 497-504.

Biferale, L., Boffetta, G., Celani, A., Devenish, B. J., Lanotte, A. \& Toschi, F. 2005 Multiparticle dispesion in fully developped turbulence. Phys. Fluids 17, 111701.

Bodenschatz, E., Bewley, G. P., Nobach, H., Sinhuber, M. \& Xu, H. 2014 Variable density turbulence tunnel facility. Rev. Sci. Instr. 85, 093908.

Borue, V. \& Orszag, S. A. 1998 Local energy flux and subgrid-scale statistics in threedimensional turbulence. J. Fluid Mech. 336, 1-31.

Buaria, D., Pumir, A., Bodenschatz, E. \& Yeung, P. K. 2019 Extreme velocity gradients in turbulent flows. New J. Phys. 21, 043004.

Carbone, M. \& BragG, A. D. 2020 Is vortex stretching the main cause of the turbulent energy cascade? J. Fluid Mech. 883, R2.

Chertkov, M., Pumir, A. \& Shraiman, B. I. 1999 Lagrangian tetrad dynamics and the phenomenology of turbulence. Phys. Fluids 11, 2394-2410.

Chevillard, L. \& Meneveau, C. 2006 Lagrangian dynamics and statistical geometric structure of turbulence. Phys. Rev. Lett. 97, 174501.

Comte-Bellot, G. \& Corrsin, S. 1966 The use of a contraction to improve the isotropy of grid-generated turbulence. J. Fluid Mech. 25, 657.

Devenish, B. J. 2013 Geometrical properties of turbulent dispersion. Phys. Rev. Lett. 110, 064504.

Douady, S., Couder, Y. \& Brachet, M. E. 1991 Direct observation of the intermittency of intense vorticity filaments in turbulence. Phys. Rev. Lett. 67, 983-986.

FRISCH, U. 1995 Turbulence: The Legacy of A. N. Kolmogorov. Cambridge, England: Cambridge University Press.

Hackl, J. F., Yeung, P. K. \& Sawford, B. L. 2011 Multi-particle and tetrad statistics in numerical simulations of turbulent relative dispersion. Phys. Fluids 23 (6), 065103.

Ishinara, T., Kaneda, Y., Yosokawa, M., Itakura, K. \& A.Uno 2007 Small-scale statistics in high resolution of numerically isotropic turbulence. J. Fluid Mech. 592, 335-366.

Jimenez, J., Wray, A. A., Saffman, P. G. \& Rogallo, R. S. 1992 The structure of intense vorticity in isotropic turbulence. J. Fluid Mech. 255, 65-90.

Johnson, P. L. \& Meneveau, C. 2016 A closure for lagrangian velocity gradient evolution in turbulence using recent deformation mapping of initilly gaussian field. J. Fluid Mech. 804, $387-419$.

Johnson, P. L. \& Meneveau, C. 2018 Predicting viscous-range velocity gradient dynamics in large-eddy simulations of turbulence. J. Fluid Mech. 837, 80-114.

Jucha, J., Xu, H., Pumir, A. \& Bodenschatz, E. 2014 Time-reversal-symmetry breaking in turbulence. Phys. Rev. Lett. 113, 054501.

von Kármán, T. \& Howarth, L. 1938 On the statistical theory of isotropic turbulence. Proc. Roy. Soc. Lond. A 164, 192-215.

Li, Y., Perlman, E., Wan, M., Yang, Y., Meneveau, C., Burns, R., Chen, S., Szalay, A. \& EYINK, G. L. 2008 A public turbulence database cluster and applications to study lagrangian evolution of velocity increments in turbulence. Journal of Turbulence 9 (N31), $1-29$.

Lüthi, B., Оtт, S., Berg, J. \& Mann, J. 2007 Lagrangian multi-particle statistics. J. Turbul. 8,45 .

Meneveau, C. 2011 Lagrangian dynamics and models of the velocity gradient tensor in turbulent flows. Annu. Rev. Fluid Mech. 43, 219-245.

Meneveau, C. \& Lund, T. S. 1994 On the lagrangian nature of the turbulence energy cascade. Phys. Fluids 6, 2820-2825.

Monin, A. S. \& Yaglom, A. M. 1975 Statistical Fluid Mechanics. Cambridge University Press. 
NAso, A. 2019 Multiscale analysis of the structure of homogeneous turbulence. Phys. Rev. Fluids 4, 024609 .

Naso, A. \& Godeferd, F. S. 2012 Statistics of the perceived velocity gradient tensor in a rotating turbulent flow. New J. Phys. 14, 125002.

NAso, A. \& Pumir, A. 2005 Scale dependence of the coarse-grained velocity derivative tensor structure in turbulence. Phys. Rev. E 72, 056318.

Pope, S. B. 2000 Turbulent flows. Cambridge University Press.

Pumir, A., Bodenschatz, E. \& Xu, H. 2013 Tetrahedron deformation and alignment of perceived vorticity and strain in a turbulent flow. Phys. Fluids 25, 035101-.

Pumir, A., Shraiman, B. I. \& Chertkov, M. 2000 Geometry of lagrangian dispersion in turbulence. Phys. Rev. Lett. 85 (25), 5324-5327.

SigGia, E. 1981 Numerical study of small-scale intermittency in three-dimensional turbulence. J. Fluid Mech. 107, 357-406.

Tao, B., Katz, J. \& Meneveau, C. 2002 Statistical geometry of subgrid-scale stresses determined from holographic particle image velocimetry measurements. J. Fluid Mech. 457, $35-78$.

Townsend, A. A. 1951 On the fine-scale structure of turbulence. Proc. Roy. Soc. A 208, 534542.

Tsinober, A. 2009 An Informal Conceptual Introduction to Turbulence. Berlin: Springer.

Van der Bos, F., TaO, B., Meneveau, C. \& Katz, J. 2002 Effects of small-scale turbulent motions on the filtered velocity gradient tensor as deduced from holographic piv measurements. Phys. Fluids 14, 2456-74.

Xu, H., Ouellette, N. T. \& Bodenschatz, E. 2008 Evolution of geometric structures in intense turbulence. New J. Phys. 10, 013012-.

Xu, H., Pumir, A. \& Bodenschatz, E. 2011 The pirouette effect in turbulent flows. Nat. Phys. 7, 709-712.

Xu, H., Pumir, A. \& Bodenschatz, E. 2016 Lagrangian view of time irreversibility of fluid turbulence. Sci. China-Phys. Mech. Astron. 59 (1), 614702-614702.

Yeung, P. K., Donzis, D. A. \& Sreenivasan, K. R. 2012 Dissipation, enstrophy and pressure statistics in turbulence simulations at high reynolds numbers. J. Fluid Mech. 700, 5-15. 